\title{
Underwater Wireless Acousto-Optic Waveguide (UWAOW)
}

Giovanni Giuliano, Lionel W. J. Kent, Leslie C. Laycock

Giovanni Giuliano, Lionel W. J. Kent, Leslie C. Laycock, "Underwater Wireless Acousto-Optic Waveguide (UWAOW)," Proc. SPIE 10437, Advanced Free-Space Optical Communication Techniques and Applications III, 1043708 (6 October 2017); doi: 10.1117/12.2286318

SPIE. Event: SPIE Security + Defence, 2017, Warsaw, Poland 


\title{
Underwater Wireless Acousto-Optic Waveguide (UWAOW)
}

\author{
Giovanni Giuliano ${ }^{\mathrm{ab}}$, Lionel W. J. Kent ${ }^{\mathrm{b}}$, and Leslie C. Laycock ${ }^{\mathrm{b}}$

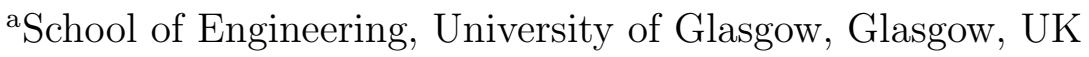 \\ ${ }^{\mathrm{b}} \mathrm{BAE}$ Systems Applied Intelligence, Chelmsford Technology Park, Chelmsford, UK
}

\begin{abstract}
The present study originated in the lack of research into achieving underwater total internal reflection (TIR) via the acousto-optic effect. The uniqueness of this technique exists in the fact that it is based on a high sound pressure level which induces a localised change in refractive index of seawater sufficient to achieve total internal reflection within the communication channel. Different transducer systems for generating the pressure wave have been investigated and take the form of a wave which may be either a standing wave, or a novel beamforming technique. The former is based on an array of transducers and with an acoustic mirror at the receiver in order to establish the standing wave. The alternative approach relies on the high intrinsic directionality of a novel beamformer where an annular transducer array is examined as an acoustic source. In this paper, the main characteristics of the acoustic optic waveguide will be presented. This will include both sound and light propagation in the ocean, TIR, novel beam propagation, the refractive index of water as a function of the externally applied acoustic pressure, and the acoustic technology. The modelled results, the limitations imposed by the challenging medium, and the system requirements required to obtain an Underwater Wireless AcoustoOptic Waveguide (UWAOW) will be also addressed.
\end{abstract}

Keywords: acousto-optic effect, free-space laser communication, waveguiding, total internal reflection, transducer, beamforming technique, ocean optics

\section{INTRODUCTION}

During recent years considerable progress has been made in the field of underwater optical communications given the scientific and military interest in their research and application. A novel technique is described for laser based underwater communication systems. Electromagnetic waves that fall in the blue-green region of the visible spectrum have received the most interest since they correspond to the minimum of optical attenuation within the medium. Nevertheless, the optical transmission losses of seawater range from $0.1 \mathrm{~dB} \mathrm{~m}^{-1}$ in clear ocean waters to several $\mathrm{dB} \mathrm{m}^{-1}$ in a harbour, as shown in Table 1 . These values impose severe limitations on the maximum communication distance for the system. This is because optical power decreases exponentially with the distance from the laser source, and it is dominated by the total extinction coefficient $\left(c\right.$, in $\left.\mathrm{m}^{-1}\right)$ which is the sum of both absorption $(a)$ and scattering $(b)$ by the seawater. These, in turn, are Inherent Optical Properties (IOPs) and thus they are dependent only upon the medium. For this reason, it is not possible to overcome the exponential attenuation of seawater.

\begin{tabular}{|c|c|c|c|}
\hline Water type & $\mathbf{c}\left[\mathbf{m}^{-1}\right]$ & $c\left[\mathrm{~dB} \mathrm{~m}^{-1}\right]$ & $-10 \mathrm{~dB}$ distance $[\mathrm{m}]$ \\
\hline Pure sea & 0.043 & -0.19 & 53.55 \\
\hline Clear ocean & 0.151 & -0.66 & 15.25 \\
\hline Coastal ocean & 0.399 & -1.73 & 5.77 \\
\hline Harbour water & 2.195 & -9.53 & 1.05 \\
\hline
\end{tabular}

Table $\overline{\overline{1: \text { Optical attenuation values in four typical oceanic water types at } 530}} \mathrm{~nm} .{ }^{1}$

In this work, the resulting change in refractive index resulting from an external acoustic field is referred to as an Underwater Wireless Acousto-Optic Waveguide (UWAOW). The technique described aims to minimise

Further author information: Giovanni Giuliano, g.giuliano.1@research.gla.ac.uk 
the adverse effect of scattering within the communication channel. Scattering losses are one of the current technical challenges associated with any underwater free-space communication due to the inherent properties of the medium. The main expected benefit arising from the implementation of the proposed novel technique is minimised photon time of flight dispersion, that would result in an augmented collected optical power at the photoreceiver. This, in turn, would increase the electrical signal-to-noise ratio that is directly related to the data throughput and the maximum achievable range under varying conditions. A novel technique based on a simple but powerful concept is here described. We targeted our research on two different embodiments in order to efficiently establish and maintain a free-space wave-guiding along the optical communication channel. In this work we present a comparison of their advantages and disadvantages, and we provide numerical examples for their implementation. Ongoing work will further develop this model.

In the following sections the main characteristics of underwater acoustic propagation will be presented: sound and light propagation in the ocean, TIR, Bessel beam, refractive index of water as function of the externally applied acoustic pressure, and the SONAR technology. Then the results, the limitation imposed by the challenging medium, and the system requirements required to obtain an UWAOW will be discussed. An extensive and more detailed discussion of each topic has not been provided here since it can be found in the related literature.

\section{BACKGROUND THEORY}

Acoustic waves are well known to propagate better through water than in air, at least since the 15th century when Leonardo da Vinci observed that "If you cause your ship to stop and place the head of a long tube in the water and place the outer extremity to your ear, you will hear ships at a great distance from you.". ${ }^{2}$ Acoustic waves have been used worldwide in the underwater environment since World War I for many applications and they still represent, in many scenarios, the most efficient technique to communicate at long distances, although at relatively low data rates due to the limited bandwidth. The considerable amount of research that has been carried out over the past decades represents a resource and a know-how that might be potentially transferred and applied to an underwater optical communication system (UOCS). To the authors best knowledge, previous studies on the change in refractive index of water with an external acoustic field only focussed on measuring techniques such as the non-intrusive Laser Doppler Anemometry (LDA) ${ }^{3}$ and laser vibrometry. ${ }^{4}$ The LDA technique has been widely used to measure the instantaneous acoustic particle velocity in fluids. ${ }^{5-7}$

Here we aim to control the refractive index of seawater to form a waveguide to limit scattering. There are a number of potential ways to achieve this. With reference to seawater, which is a diamagnetic substance with a variable concentration of dissolved salt, there have been a number of studies on the magnetic effect on its refractive index. When using high magnetic fields (10 T, almost 400000 times the Earth's magnetic field) it is has been observed that water can levitate in $\operatorname{air}^{8}$ and that there is an increase in the refractive index up to $0.1 \%,{ }^{9}$ an effect that is attributed to an increased hydrogen bond strength. The reasons why a magnetic field is not studied in this report are its difficulty in being transferred underwater, and the fact that the resulting change in the refractive index is relatively small compared that required to realise a waveguide channel. As briefly discussed above, there are two main IOP. The first is absorption which is caused by in-suspension matter, colour dissolved organic matter (CDOM) and chlorophyll. Their concentration within the water column are the main factors that contribute to the final value of absorption and thereby the total extinction coefficient $c$. The second IOP is scattering of incident photons into other directions that reduces the forward on-axis transmission. This gives an angular redistribution of the optical field (i.e. beam spreading) that results in lost optical power if the receiver aperture is not large enough to collect it. This study consider the containment of beam spreading by examining the hypothesis that an optical waveguide can be obtained by the use of a high sound pressure level (SPL). The work is supported by numerical simulation based on the available data in the scientific literature.

A schematic of the basic working principle of an UWAOW is shown in Figure 1. It is based on sound waves propagating underwater along the optical communication channel in order to realise a localised increase in its refractive index. The mechanical strain in the channel due to the external acoustic field locally modifies the molecular polarizability. The effect of the acoustic pressure is to increase the refractive index of seawater from $n_{0}$ to $n_{1}$, where $n_{0}$ is the refractive index of the medium when no acoustic pressure is applied, hence $n_{1}>n_{0}$. The acoustic source, not drawn for the sake of simplicity, is located at the origin of the TIR's cone assuming a 
far-field region (as discussed in Section 3.2). This sound field expands through the communication channel due to the geometrical spreading and support TIR up to a distance where the sound field intensity is still sufficient to induce the required change in the refractive index needed to support TIR. In the following sections, the basic description of the ocean acoustic and optical fields that lead to the proposed UWAOW are presented.

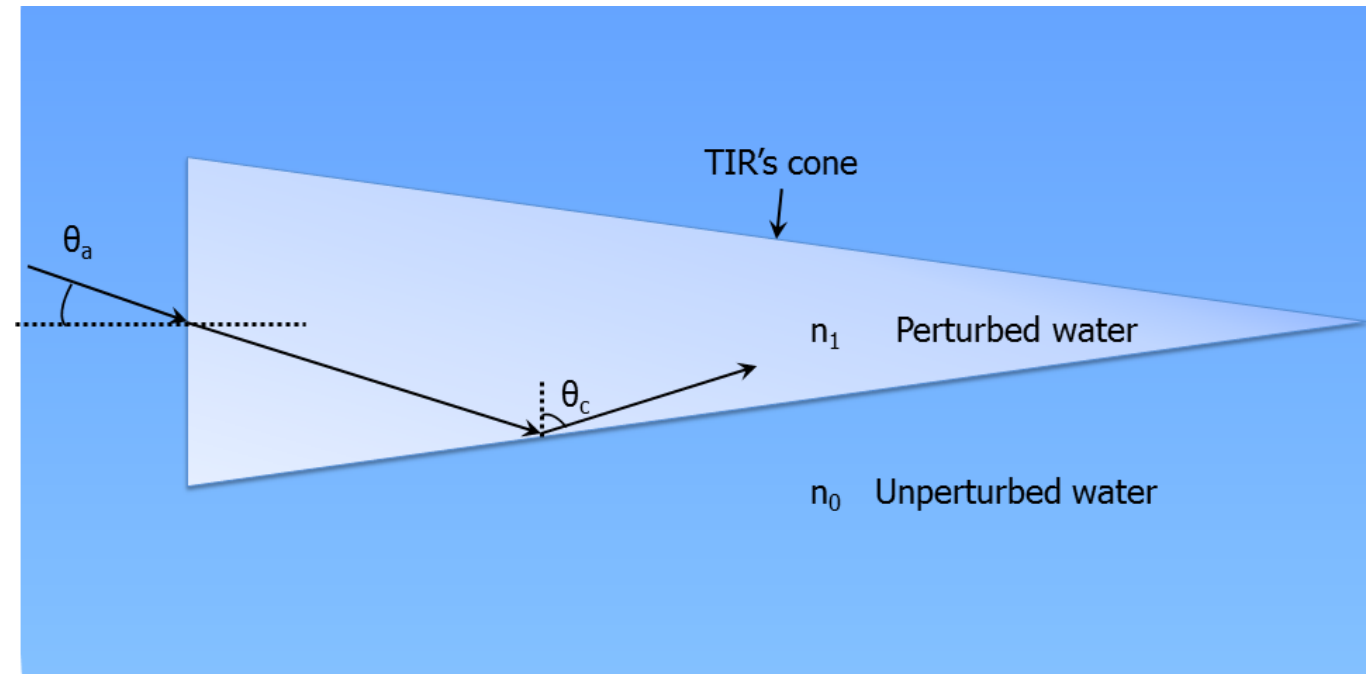

Figure 1: Illustration of the proposed UWAOW with an incident optical ray at the maximum half acceptance angle $\theta_{a}$ of the waveguide with a critical angle $\theta_{c}$ for TIR.

\subsection{Sound absorption in seawater}

The frequency dependence of sound absorption has been widely studied in the past and many equations are available in the scientific literature. It is anticipated that in the scenario considered here, characterised by a relatively short propagation range $(<100 \mathrm{~m})$ and acoustic frequencies of less than $100 \mathrm{kHz}$, the impact of acoustic absorption is negligible. A simplified expression for the sound absorption $\alpha^{\prime}$ expressed in $\mathrm{dB} \mathrm{km}^{-1}$ and valid for a salinity $S=35 \mathrm{ppt}$, at a temperature $T=4{ }^{\circ} \mathrm{C}$ and $p H=8$ was derived by Fisher \& Simmons (with $f$ expressed in $\mathrm{kHz})^{2,10,11}$

$$
\alpha^{\prime}=3.3 \times 10^{-3}+\frac{0.11 f^{2}}{1+f^{2}}+\frac{44 f^{2}}{4100+f^{2}}+3.0 \times 10^{-4} f^{2}
$$

A few values for $\alpha^{\prime}$ are calculated and summarised in Table 2. It can be seen that there are different regions in the frequency dependence of absorption due to the different dominant processes: chemical relaxation in the frequency range $0.1 \mathrm{kHz}$ to $100 \mathrm{kHz}$, and shear and bulk viscosity for $f>100 \mathrm{kHz} .^{11}$ It is worth noting that the absorption for acoustic waves $\alpha^{\prime}$ is expressed as $\mathrm{dB} \mathrm{km}^{-1}$. When referring to the extinction coefficient in the optical domain, $c$, this is usually given in $\mathrm{dB} \mathrm{m}^{-1}$ as seawater more strongly absorbs EM waves (see Table 1), although with an optical window in the blue-green region of the spectrum $(450 \mathrm{~nm}$ to $550 \mathrm{~nm})$. Therefore in this report underwater free-space optical links up to $30 \mathrm{~m}$ with an UWAOW operating at maximum $100 \mathrm{kHz}$ are evaluated; the acoustic losses due to absorption by seawater are negligible in the acoustic range $0.02 \mathrm{kHz}$ to $20 \mathrm{kHz}$ whereas they have a greater impact on the link budget as the acoustic frequency is increased.

\subsection{Speed of sound underwater}

The first parameter considered is the speed of sound underwater $c_{w}$. This value underwater is widely variable and many equations have been proposed in the literature. In this work the recent formulation proposed by Leroy et al. ${ }^{12}$ in 2008 (that is accurate within $\pm 0.2 \mathrm{~m} \mathrm{~s}^{-1}$ ), valid in all oceans, is used. with temperature $T$ in ${ }^{\circ} \mathrm{C}$ (1990 universal temperature scale), salinity $S$ in \%o (i.e. in parts per thousand), depth $z_{d}$ in m, and latitude 


\begin{tabular}{c|c|c}
\hline \hline Frequency $[\mathrm{kHz}]$ & $\alpha^{\prime}\left[\mathbf{d B} \mathbf{k m}^{-1}\right]$ & $\mathbf{- 6} \mathbf{~ d B}$ distance $[\mathrm{km}]^{*}$ \\
\hline 0.1 & 0.0045 & 1333.503 \\
\hline 1.0 & 0.0693 & 86.544 \\
\hline 10.0 & 1.1898 & 5.043 \\
\hline 100.0 & 34.3190 & 0.175 \\
\hline 1000.0 & 343.9336 & 0.017 \\
\hline \hline
\end{tabular}

Table 2: Summary of sound absorption values in seawater and -6 dB distance from the acoustic source as per Equation (1). * Ignoring acoustic spreading losses.
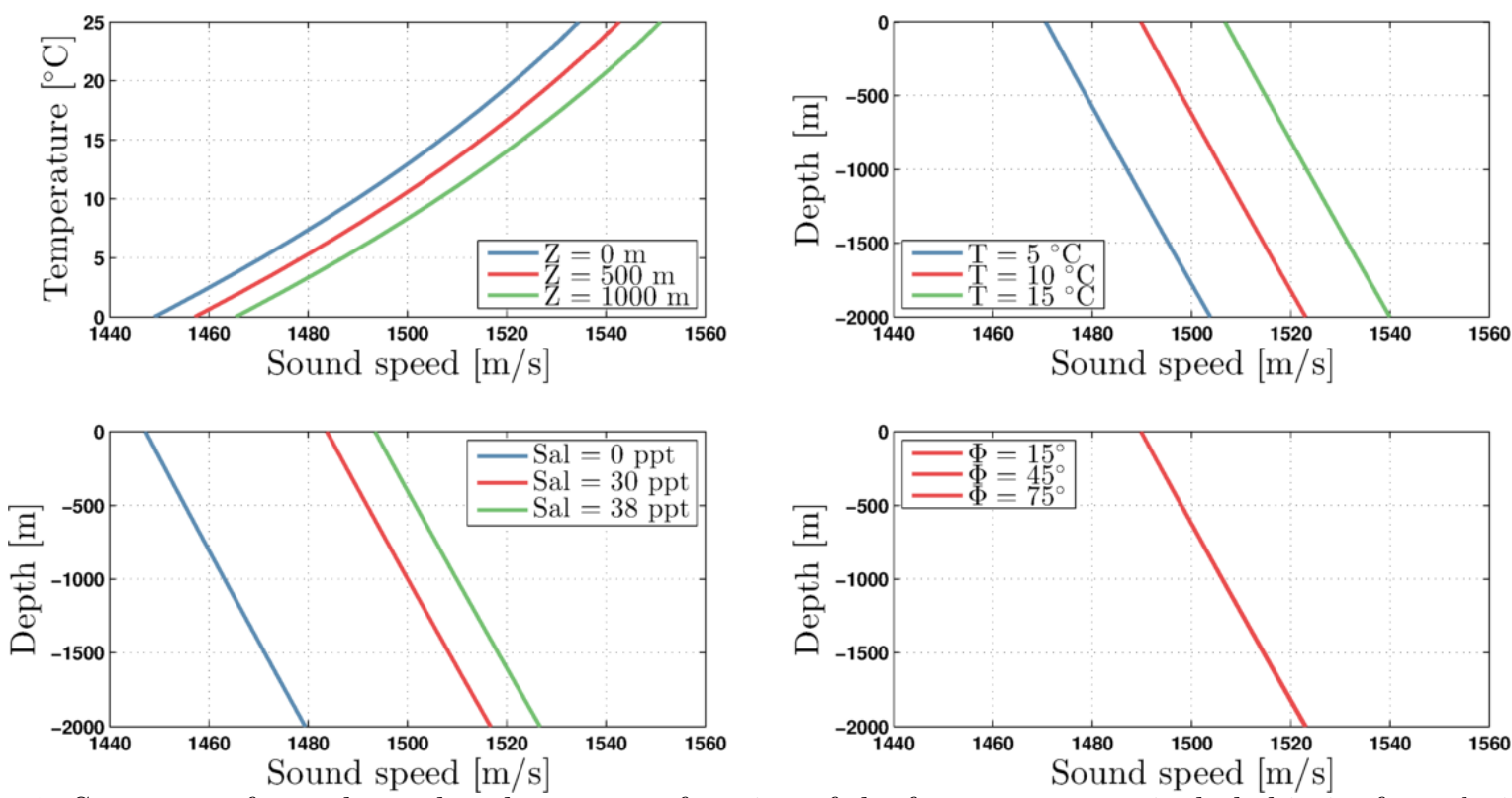

Figure 2: Summary of sound speed underwater as function of the four parameters included in its formulation by Leroy et al. ${ }^{12}$ The fixed values for the parameters in each subplot, except where otherwise specified, are: $T=$ $10^{\circ} \mathrm{C}, z_{d}=100 \mathrm{~m}, S=35 \mathrm{ppt}$ and $\Phi=45^{\circ}$.

$\Phi$ in degrees. Comparative plots illustrating the influence of the four parameters are shown in Figure 2, with likely combinations of salinity, latitude, depth and temperature. The scale on the $x$-axis is kept constant for all of the four plots in order to offer an easier comparison between them. Across the world salinity levels vary from $0 \%$ for fresh water to levels as high as $38 \%$ in the Mediterranean Sea. The influence of latitude on the sound speed value can be neglected since its value at different latitudes remains approximately constant up to a certain degree of accuracy $\left( \pm 0.1 \mathrm{~m} \mathrm{~s}^{-1}\right.$ between two curves). From an analysis of these plots, a reasonable choice for an approximate average speed of sound underwater is $c_{w}=1500 \mathrm{~m} \mathrm{~s}^{-1}$ when setting the simulation parameters for the modelling of a real underwater scenario. In this study an isovelocity water channel is assumed: it should however be noted that the exact sound speed for any different scenario can be obtained by using the equation proposed by Leroy et al. ${ }^{12}$ if the system is likely to working within an environment described by a different range of values that would result in a sound speed that differs from the reference value by a significant degree.

\subsection{Light attenuation in seawater}

The optical properties of seawater are highly variable, depending on various factors such as depth, CDOM and time of the day, among others. An approximate classification can be done based on the average indicative values of the IOPs and results in a four water types as identified by C. Mobley: ${ }^{1}$ pure sea, clear ocean, coastal ocean and harbour water. The most likely application of this technique may be applied in harbour and coastal waters where the scattering plays the main role in the overall beam attenuation. Indeed, an UWAOW can be also 
seen as a communication channel with a higher water clarity thanks to the effect of the high sound pressure applied. In order to evaluate the paramount importance of reduced scattering in the context of UOCS, an useful oceanic parameter is the single-scattering albedo of the water, defined as $w_{a}=b / c$, which is the ratio of scattering loss to total loss. As it would be expected, in harbour and coastal waters a photon is more likely to be scattered, thus $w_{0}$ is closer to one. Table 3 depicts the absorption $(a)$, scattering $(b)$, extinction coefficient $(c)$ and single-scattering albedo $\left(w_{a}\right)$ for four types of oceanic waters. From these data it can be noticed that the total extinction coefficient $c(\lambda)$ increases from $<0.1 \mathrm{~m}^{-1}$ in pure seawater to $>2 \mathrm{~m}^{-1}$ in turbid harbour water. It is more challenging to design a UOCS near the shore than open ocean since the absorption coefficient increases more moderately than does the turbidity, i.e the scattering.

\begin{tabular}{c|c|c|c|c}
\hline \hline Water type & $\mathbf{a}\left(\mathbf{m}^{-\mathbf{1}}\right)$ & $\mathbf{b}\left(\mathbf{m}^{-\mathbf{1}}\right)$ & $\mathbf{c}\left(\mathbf{m}^{-\mathbf{1}}\right)$ & $\mathbf{w}_{a}$ \\
\hline Pure sea & 0.0405 & 0.0025 & 0.043 & 0.006 \\
\hline Clear ocean & 0.114 & 0.037 & 0.151 & 0.247 \\
\hline Coastal ocean & 0.179 & 0.220 & 0.399 & 0.551 \\
\hline Harbour water & 0.366 & 1.829 & 2.195 & 0.833 \\
\hline \hline
\end{tabular}

Table 3: Typical optical properties of four different ocean water types at $530 \mathrm{~nm} .^{1}$

From the data reported in the table, it is evident that a minimised scattering component would be of great help in many scenarios which involves underwater operations based in harbour and coastal waters. In the following sections, the necessary tools to design and develop an UWAOW will be discussed.

\subsection{Total Internal Reflection (TIR)}

It is well known that, in an optical fibre, the higher refractive index of the core $\left(n_{1}\right)$ with respect to the cladding $\left(n_{0}\right)$ results in the reflection of light that as a consequence remains confined within the core if the angle of the incident ray is within the acceptance angle. This phenomenon is referred to as total internal reflection (TIR) and it permits the possibility to guide and transmit the light (i.e. the information encoded into it) over long distances $(>100 \mathrm{~km})$. The order of magnitude for free-space propagation of acoustic waves underwater is comparable with this range as a result of the low absorption coefficient of water $\left(<1 \mathrm{~dB} \mathrm{~km}^{-1}\right)$ for frequencies up to $1 \mathrm{kHz}$ (Table 2). The main drawback of underwater acoustic communication is the limited channel bandwidth which limits the maximum data rate many to orders of magnitude lower than optical communication which can achieve Gbps throughput. ${ }^{13}$ The maximum bandwidth arises from an intrinsic limitation given by the carrier frequency and the Nyquist rate, in addition to various contingencies in a real scenario. Following the analogy with an optical fibre, it is useful to describe the refractive index contrast $\Delta$ between perturbed water $\left(n_{1}\right)$ and unperturbed water $\left(n_{0}\right)$, expressed in $\%$, as

$$
\Delta=100\left(\frac{n_{1}^{2}-n_{0}^{2}}{2 n_{1}^{2}}\right) .
$$

Both the numerical aperture (NA) of the waveguide and the half acceptance angle $\theta_{a}$ (see Figure 1) increase with $\Delta$ and they are given by

$$
N A=n_{0} \sin \left(\theta_{a}\right)=\sqrt{n_{1}^{2}-n_{0}^{2}}
$$

The higher the NA, the easier it is to couple between the laser beam and the waveguide as a result of the wider acceptance angle at which the incident light can enter the UWAOW.

\subsection{Acoustic Bessel beam}

A brief description of the acoustic Bessel beam properties is here described that will serve as a basis for one of the two proposed UWAOW geometry later on. One particular solution of the free space wave equation

$$
\left(\nabla^{2}-\frac{1}{c^{2}} \frac{\partial^{2}}{\partial t^{2}}\right) U(\mathbf{r}, t)=0
$$


for a wave with amplitude $U(\mathbf{r}, t)$ was proposed by J. Durnin in $1987^{14}$ by using a Bessel function (of the first kind) of the zeroth-order $J_{0}(\alpha \rho)$ as

$$
U(\mathbf{r}, t)=J_{0}(\alpha \rho) \exp [i(\beta z-\omega t)],
$$

where $\rho=\sqrt{x^{2}+y^{2}}$ is the radial distance from the transducer centre which is characterized by two real parameters, $\alpha$ and $\beta$ having SI units of reciprocal of meter. These, in turns, are related to the wavenumber $k$ and the angular frequency $\omega$ by the condition

$$
\alpha^{2}+\beta^{2}=k^{2}=\left(\frac{2 \pi}{\lambda_{a c}}\right)^{2}=\left(\frac{\omega}{c_{w}}\right)^{2} .
$$

The most striking property of a resulting Bessel beam based on a Bessel function relies on its propagationinvariant nature, that arises from a mathematical consideration based on its independence from the propagation in the $z$ direction. Thus, since the cross-sectional energy remains constant (excluding losses other than spreading), in principle this type of beam would contain the same amount of energy in the central lobe (core) and in each sidelobe (rings). ${ }^{14,15}$ In particular, $J_{0}(\alpha \rho)$ has the best ratio between central lobe and sidelobes, and thus it has received much interest in the studies of the approximation of a Bessel beam. In practice, Bessel-like beams with a finite propagation distance were experimentally realised by use of a narrow annular slit, ${ }^{14,16}$ or by a conically shaped element (axicon) ${ }^{17}$ or by using an annular transducer array. ${ }^{18-20}$ Thanks to their narrow central core that enhances the lateral resolution and their longer propagation distance respect to conventional Gaussian beams, the applications of these propagation-invariant beams span from medical imaging systems ${ }^{18}$ to atom optics. ${ }^{21}$ In the context of their application in an UWAOW, the main characteristics of an annular transducer array, the choice of the optimal parameters value to approximate a Bessel beam and how to implement the fundamental advantage of its well-collimated central core will be described in Section 3.4.

\subsection{Underwater acoustic parameters}

In this section, the fundamentals of underwater acoustics and the relevant units are briefly discussed before presenting the results. The root-mean-squared intensity of any plane wave is given by the pressure, $P$, and the acoustic impedance $Z .{ }^{11}$ In underwater acoustics, given the high dynamic range of pressure values, the quantities are expressed in $\mathrm{dB}$ using as pressure reference $P_{0}=1 \mu \mathrm{Pa}$. The SPL in air is referenced to $20 \mu \mathrm{Pa}$, thus the same acoustic field above and under water will be higher by $26 \mathrm{~dB}$ in the latter. In addition to this, the lower acoustic impedance of air results in

$$
P_{w} / P_{a i r}=20 \log _{10} \sqrt{Z_{w} / Z_{a i r}}=20 \log _{10} \sqrt{\left(\rho_{w} c_{w}\right) /\left(\rho_{\text {air }} c_{\text {air }}\right)}=35.64 \mathrm{~dB}
$$

thus approximately an additional $36 \mathrm{~dB}$ of difference. It follows that when considering the same acoustic intensity in air and underwater it is necessary to subtract approximately $62 \mathrm{~dB}$ from the second value to avoid confusion and misunderstandings. In addition to this, and due to the big difference in acoustic impedances $Z_{w}$ and $Z_{\text {air }}$, there is negligible transmission of sound from underwater to above water since the air-water interface works as an almost perfect reflector (Lloyd mirror). ${ }^{22}$ It is important to note that there is a significant difference in the acoustic impedance between the transducers core and the medium, i.e. seawater in this work. In order to maximise the acoustic transmission from the transducer's surface to the seawater, it is necessary to choose an intermediate layer that will match the impedance of the former with the latter. The optimal thickness of the coating layer has the be one quarter of the designed operating acoustic wavelength in order to maintain the reflected waves in phase at the output of the matching layer. For example, if $f=100 \mathrm{kHz}, \lambda_{a c}=0.015 \mathrm{~m}$, hence the thickness of the coating layer would be approximately $4 \mathrm{~mm}$. As discussed above, the approximate average acoustic impedance for seawater is $Z_{w}=\rho c_{w}=1.5$ MRayl. The most commonly material used by manufacturers for transducers is stainless steel which has a typical value of $Z_{s s}=45$ MRayl. Therefore, transmission losses will be minimised with a coating layer medium that has an ideal acoustic impedance of

$$
Z_{\text {layer }}=\sqrt{Z_{s s} Z_{w}}=7.7 \text { MRayl. }
$$

Thus a suitable material with an acoustic impedance close to 7.7 MRayl would optimise the performances of the coated transducer system. Recent research in this field has shown evidence that a suitable choice could be 
suitably tailored alumina colloidal/polymer nanocomposite films. ${ }^{23}$ For example, a resulting acoustic impedance of 5.1 MRayl was measured with an alumina fraction of $32 \%{ }^{24}$

A second consideration is acoustic propagation: acoustic waves in fluids are longitudinal compression waves since fluids cannot support transverse polarised waves. The density change is given by molecules that will return to their original position without a net flow of water within the acoustic channel. Solids, on the contrary, are able to support both longitudinal and transverse waves. The acousto-optic effect results from the increased density of water exerted by the acoustic pressure. The commonly held view that fluids are incompressible is true up to a certain approximation. Indeed even if their change in volume (thus in their density) is very small, it can start to become evident at high levels of applied pressure.

\subsection{Influence of acoustic pressure on the refractive index of water}

A change in the refractive index of materials occurs in different situations, particularly when high intensities are applied. For example, the Pockels effect as well as the Kerr effect are electro-optic phenomenons which result in, respectively, a linear and quadratic anisotropic change (birefringence) in the index of refraction in response to the external electric field applied. In this study, the influence of the acoustic pressure on the refractive index of water is investigated as a tool to achieve TIR within the communication channel. The influence of optical wavelength, temperature and density on the refractive index of water has been extensively covered by a number of research groups. More recent publications include: Schiebener et al. in 1990, ${ }^{25}$ Jack et al. in $1998,{ }^{3}$ Buick et al. in $2004^{4}$ and Weiss et al. in 2012. ${ }^{26}$ In chronological order, a comprehensive review on the refractive index of light water and steam is given by the widely cited paper of Schiebener et al. ${ }^{25}$ This formulation has also been adpted by the International Association for the Properties of Water and Steam (IAPWS) and thus it represents the widely referenced source when dealing with the refractive index of water. ${ }^{27}$ In this work, the coefficients published in 1997 by the IAPWS with the updated ITS-90 Temperature scale, are considered to evaluate the refractive index as a function of different parameters. Buick et al., ${ }^{4}$ following the model discussed by Jack et al. ${ }^{3}$ give the relation between the change in pressure (expressed in $\mathrm{Pa}$ with respect to the ambient pressure value) and the change in the refractive index in relation to the medium when no acoustic pressure is applied. Assuming a first order approximation, the equation used is

$$
\Delta P=2 \sqrt{\frac{\rho c}{M_{2}}} \Delta n
$$

Here $\rho=1027 \mathrm{~kg} \mathrm{~m}^{-3}$ is the density of water (with a salinity of $S=35 \mathrm{ppt}$ ), $c_{w}=1500 \mathrm{~m} \mathrm{~s}^{-1}$ is the speed of sound (as described in Section 2.2) and $M_{2}=1.6 \times 10^{-13} \mathrm{~s}^{3} \mathrm{~kg}^{-1}$ is the material parameter that describes the acousto-optic figure of merit as firstly measured by Pinnow in $1970 .{ }^{28}$ The value of $M_{2}$ is small since in a fluid the pressure seeks to establish the equilibrium thus minimising the anisotropy, and a considerably high acoustic pressure level is required to increase its density. Indeed, by substituting the previous parameters in Equation (9) and rearranging for the refractive index change, leads to the rather simple formula:

$$
\Delta n=K \Delta P
$$

where the constant is $K=1.605 \times 10^{-10} \mathrm{~Pa}^{-1}$ for the specific set of parameters chosen in this work as previously discussed. Despite its apparently approximated expression, the formulation given in Equation (10) is able to follow the same trend as the more complex equation proposed by Schiebener et al. ${ }^{25}$ up to a good degree of approximation, as shown later. A third relationship between refractive index and applied pressure was derived by Weiss et al. in 2012. ${ }^{26}$ It is obtained by combining the Bradley-Tait equation, that was used also by IAPWS, with the Sellmeier dispersion coefficients that are assumed to be pressure dependent. Expanding up to a second order polynomial with three coefficients, the equation can be written as a function of the applied pressure $P$ and the optical wavelength $\lambda_{o p}{ }^{26}$

The simulations plotted in Figure 3 clearly show the consistent trend in the variation of refractive index for optical wavelengths in the visible spectrum when the applied pressure is varied over many orders of magnitude. The refractive index changes more rapidly when SPL $\geqslant 260 \mathrm{~dB}$ re $1 \mathrm{\mu Pa} @ 1 \mathrm{~m}$ and its higher values at shorter wavelengths is in agreement with that reported in the previous literature. In this work, interest is focused on 


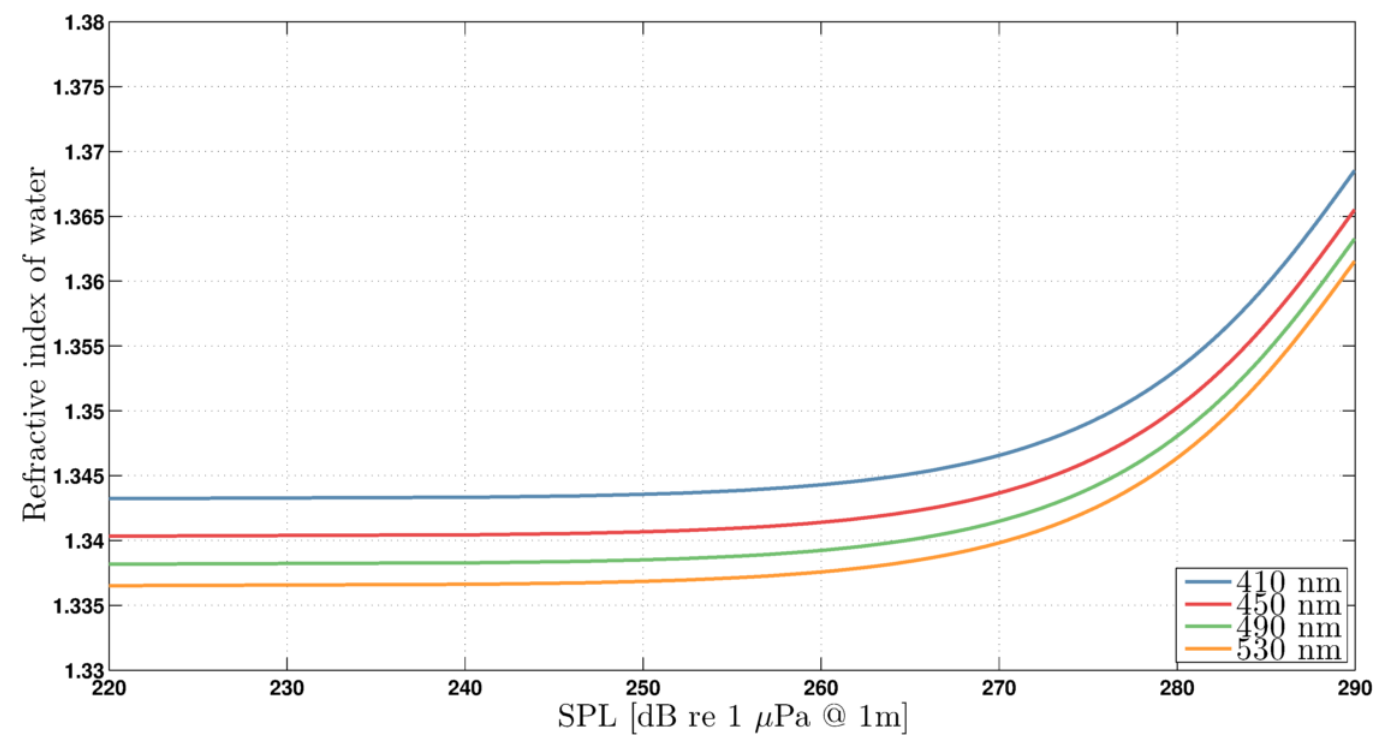

Figure 3: Influence of the acoustic pressure applied on the refractive index of water at four typical optical wavelengths usually used for free-space underwater optical communication.

the refractive index change as the applied pressure is increased to high SPL. As shown in Figure 3, the relative increment can be considered independent to the optical wavelength chosen.

The transmission wavelength $\lambda_{o p}$ is evidently an important parameter in any UOCS since it determines the absolute value for the refractive index of water, as well as its optical extinction coefficient $c$. Nevertheless, the relative increase in the refractive index of the medium is completely wavelength independent over the range of interest for an UOCS. This means that an UWAOW can be used with any type of optical beam, optical wavelength and optical power since its working principle is not linked to the optical domain but to the acoustic pressure exerted by the sound waves. In addition to this, neither the geometry of the communication link nor the atmospheric condition (such as solar background) would affect the operational capability of the proposed UWAOW that can be thus applied to any type of transmitter-receiver configuration and any time of day or night. It is now useful to compare the three relationships for the refractive index of water and the applied pressure. Figure 4 presents a summary of the three equations for a laser source operating at $589 \mathrm{~nm}$. The different trends between the relationships proposed by the three research groups are highlighted. When an optical wavelength of $589 \mathrm{~nm}$ is considered, the values at $0{ }^{\circ} \mathrm{C}$ are as listed in Table 4 . The curves are in agreement and overlap each other up to a SPL of $260 \mathrm{~dB}$ re $1 \mu \mathrm{Pa} @ 1 \mathrm{~m}$. Above this value, the formulation presented by Buick et al. ${ }^{4}$ follows the one adopted by the IAPWS, whereas the rather complicated equation suggested by Weiss et al. ${ }^{26}$ exhibits a smaller change in refractive index.

\begin{tabular}{c|c|c}
\hline \hline Pressure [MPa] & SPL $[\mathbf{d B}$ re $\mathbf{1} \mu \mathbf{P a}$ @ 1 m] & $\mathbf{n}$ \\
\hline 0.1 & 220 & 1.334344 \\
\hline 1.0 & 240 & 1.334494 \\
\hline 10.0 & 260 & 1.335969 \\
\hline 100.0 & 280 & 1.349101 \\
\hline \hline
\end{tabular}

Table 4: Refractive index of water at an optical wavelength of $589 \mathrm{~nm}$ and a temperature of $0{ }^{\circ} \mathrm{C}$ from the IAPWS formulation. $^{27}$ 


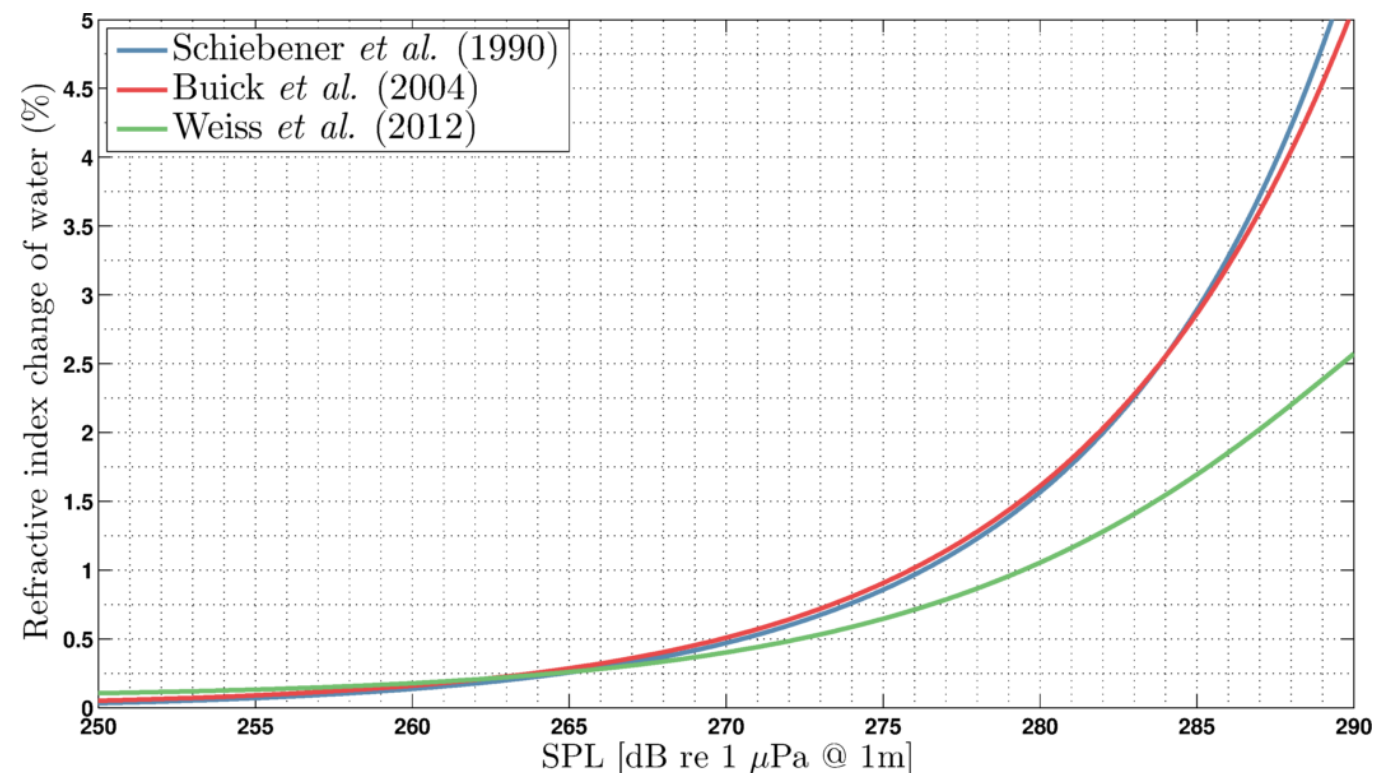

Figure 4: Change (in percent) in the refractive index of water as function of the external acoustic field.

\section{DESIGN OF AN UWAOW}

It appears from the aforementioned investigations in Section 2.4 and Section 2.7 that a considerable sound pressure is required in order to achieve a change in the refractive index of water larger than $0.1 \%$, in excess of $260 \mathrm{~dB}$ re $1 \mu \mathrm{Pa}$. The values of an hypothetical UWAOW for increasing values of SPL, starting from the minimum appreciable change of $\Delta n$, are presented in Table 5, based on Buick et al. formulation. It is well

\begin{tabular}{c|c|c|c|c|c}
\hline \hline Pressure $[\mathbf{M P a}]$ & SPL $[\mathbf{d B}$ re $\mathbf{1} \mu \mathbf{P a} @ \mathbf{1} \mathbf{~ m}]$ & $\Delta \mathbf{n}$ & $\Delta[\mathbf{\%}]$ & $\mathbf{N A}$ & $\theta_{a}\left[^{\circ}\right]$ \\
\hline 10.0 & 260 & 0.0016 & 0.12 & 0.05 & 2.8 \\
\hline 17.8 & 265 & 0.0029 & 0.21 & 0.07 & 3.8 \\
\hline 31.6 & 270 & 0.0051 & 0.38 & 0.09 & 5.0 \\
\hline 56.2 & 275 & 0.0091 & 0.67 & 0.12 & 6.7 \\
\hline 100.0 & 280 & 0.0161 & 1.19 & 0.16 & 9.0 \\
\hline 178.0 & 285 & 0.0287 & 2.08 & 0.21 & 12.0 \\
\hline 316.0 & 290 & 0.0510 & 3.61 & 0.28 & 16.2 \\
\hline \hline
\end{tabular}

Table 5: Summary of the SPL required for different UWAOW parameters. The refractive index of unperturbed water, $n_{0}$, used as reference in the calculation of $\Delta n$ and $\Delta[\%]$ is $n_{0}=1.334344$ (at $589 \mathrm{~nm}$, from $^{27}$ ).

known that in a standing wave the adjacent nodes are separated by half the acoustic wavelength with respect to the wave itself. As illustrated in Figure 5, the condition for a standing wave can be met by choosing the acoustic frequency accordingly to the length of the optical communication link and via an acoustic mirror at the photoreceiver's end.

In the following, two different geometries are proposed that are expected to fulfil the requirements for the generation of an UWAOW. The first one is based on the creation of standing wave, with an array of transducers operating at relative low frequency $(f \simeq 40 \mathrm{~Hz})$. An alternative approach relies on the high intrinsic directionality of a Bessel-like beam, as previously discussed in Section 2.5, where an annular transducer array is examined as acoustic source, operating ad ultrasonic frequencies $(f>20 \mathrm{kHz})$. 


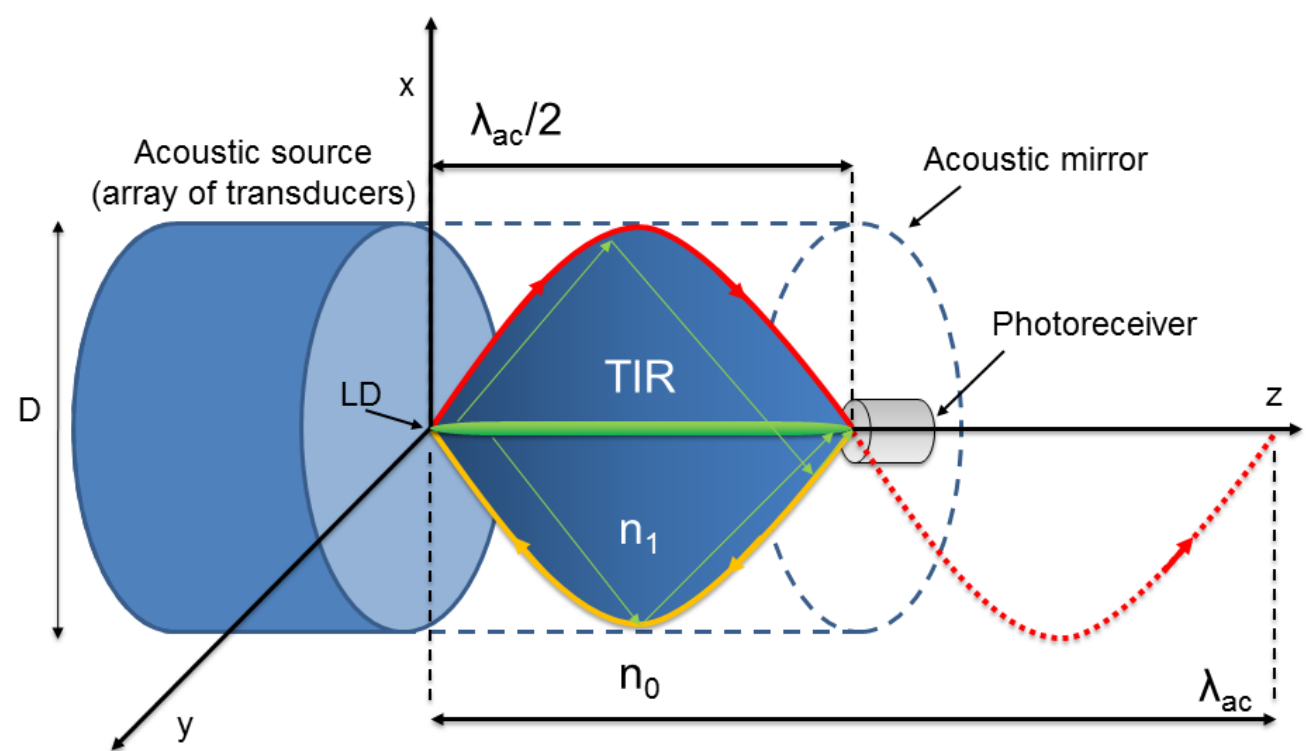

Figure 5: Schematic of the bi-directional UWAOW design that avoids nodes by achieving the condition for a standing wave formed by the forward and backward propagating acoustic waves, thus enclosing the laser beam as well as the scattered photons.

\subsection{Standing wave geometry}

The standing wave creates a waveguide with a sinusoidal profile and thus the scattered photons tends to be redirected to the center of the UWAOW where the photoreceiver aperture is centred. Hence there will be an increase in the number of collected photons and consequently also in the resulting electrical SNR at the receiver. Within this configuration, the nodes are avoided in a way that can be seen as a Bi-directional acousto-optic channel (BUWAOW). An idea of acoustic mirror (or reflector stack) is described by Epcos Ag (patent US $7230509 \mathrm{~B}^{29}$ ) made of a multiple impedance layers with a thickness of odd multiples of $\lambda_{a c} / 4$. Further details on the acoustic mirror materials and the reflector stack can be found in the patent by Agere Systems (patent US $6603241 \mathrm{~B}^{30}$ ). Thus, as the receiver is moving towards the transmitter, the acoustic frequency has to be increased in order to balance up for the shorter distance and maintain the superposition between the incident and reflected waves.

\subsection{Transmission losses}

Assuming an array of transducers with circular geometry, spherical spreading of the acoustic beam occurs in the far-field at distances greater than the so called Rayleigh distance $z_{0}:{ }^{31}$

$$
z_{0} \approx \frac{D^{2}}{2 \lambda_{a c}}
$$

where $D=2 R$ is the piston diameter of the acoustic source with radius $R$ (see Figure 5). When considering a rectangular transducer, Equation (11) is modified by dropping the factor 2 at the denominator. The fundamental reason for this difference is in their different distribution, sinc function for a square aperture and Bessel function for a circular one. In general, for a transducer of any shape, $z_{0}$ is given by the maximum dimension divided by twice the acoustic wavelength $\lambda_{a c}$. At shorter distances (near-field) the acoustic pressure and the particle velocity are not constantly in phase, resulting in a complex SPL pattern characterised by nulls and peaks as a consequence of the interference from the various sources of the acoustic array. On the contrary the SPL pattern stabilises at distances beyond $z_{0}$, once the Rayleigh distance and thus the far-field condition is reached. Transmission losses (TL) are usually modelling with two propagation regimes that describe the two extreme geometrical cases, spherical (Equation (12c) and cylindrical spreading (Equation (12a). In particular circumstances, alternative proposed models can be chosen according to the scenario by using a different value for the coefficient before the 
logarithm, such as 15 for an intermediate situation (12b) between the previous two. The equations that describe the different propagation loss regime at a distance $z$ are:

$$
T L=\left\{\begin{array}{l}
10 \log _{10}(z) \\
15 \log _{10}(z) \\
20 \log _{10}(z)
\end{array}\right.
$$

It is important to notice that sound intensity follows an inverse square law whereas pressure falls as $z^{-1}$, thus when doubling the distance, the SPL decreases by $6 \mathrm{~dB}$ with a spherical spreading beam and by only $3 \mathrm{~dB}$ for a cylindrical one. On the other side, adding two coherent equal acoustic source levels results in an increase in the SPL of $6 \mathrm{~dB}$. The different acoustic propagation regimes for various initial SPLs are shown in Figure 6 for, respectively, spherical Figure 6a and cylindrical Figure 6a spreading. As would be expected, the increase in the initial acoustic field results in a higher values throughout the entire range, with a constant separation between the curves. Indeed, even if both SPL and $T L$ are expressed in $\mathrm{dB}$, the effective SPL is simply given by their difference.

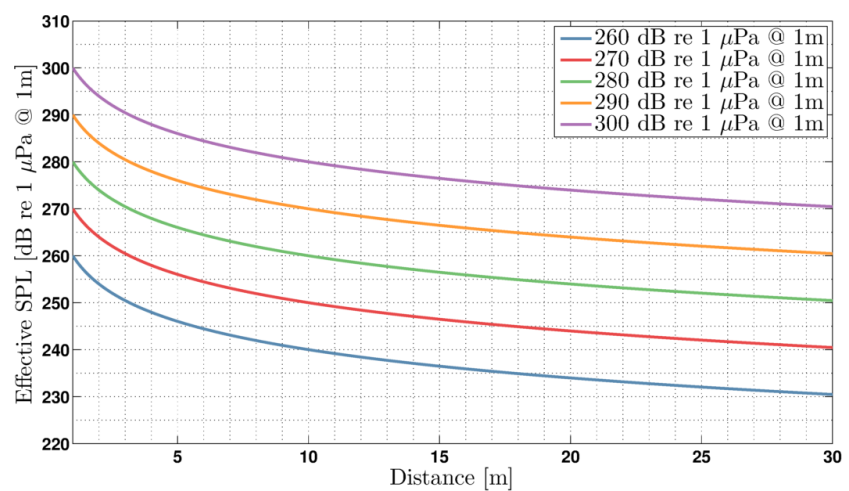

(a) Spherical spreading loss, Equation (12c)

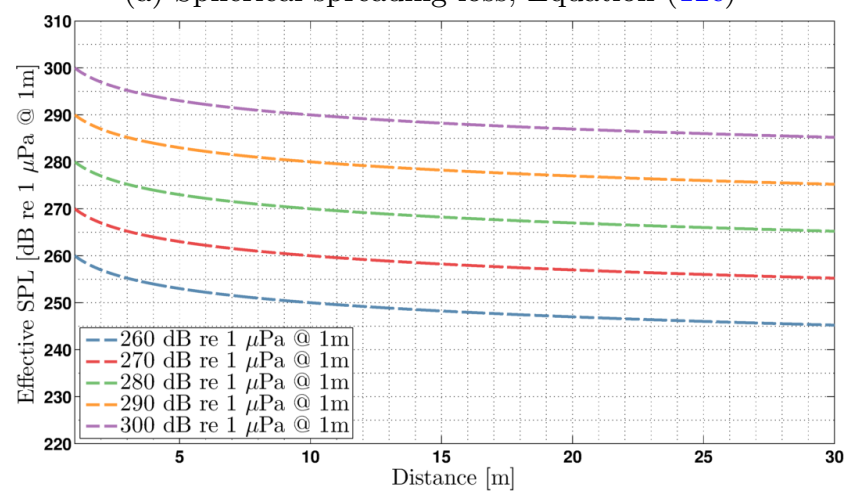

(c) Cylindrical spreading loss, Equation (12a)

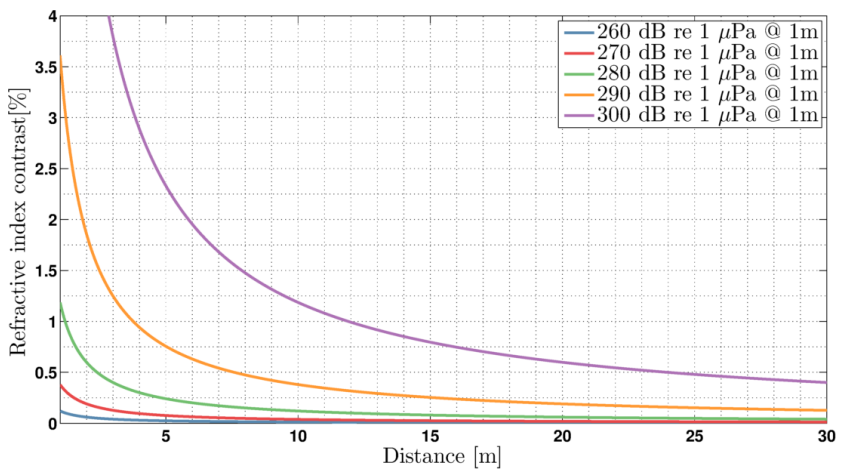

(b) Spherical spreading loss, Equation (12c)

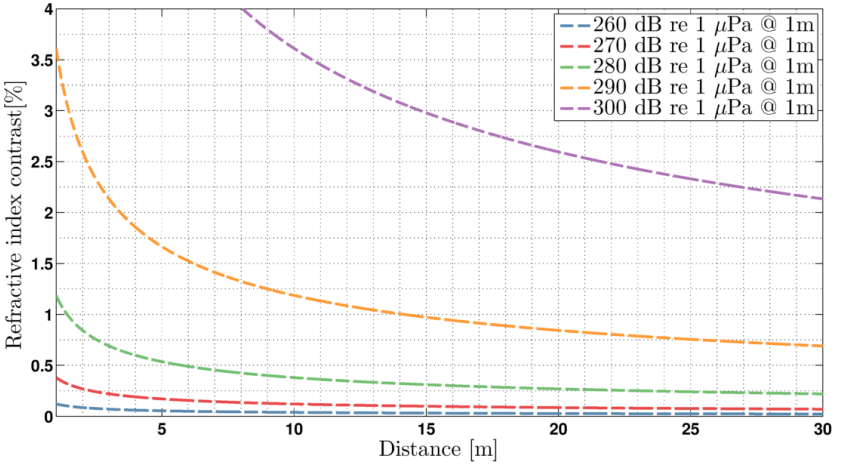

(d) Cylindrical spreading loss, Equation (12a)

Figure 6: Propagation of the acoustic field for increasing SPLs up to a distance of $30 \mathrm{~m}$ within a channel with a) spherical spreading loss and c) cylindrical spreading loss. Refractive index contrast as function of the distance from the acoustic source for different SPLs within a channel with b) spherical spreading loss and d) cylindrical spreading loss.

As previously discussed, the change in the refractive index of the medium is directly proportional to the additional external applied pressure. Thus, by combining Equation (2), Equation (10) and the specific propagation regime in Equations (12a)-(12c), the resulting effect of the acoustic field as a function of the distance from the source is presented in the left column of Figure 6. An increase in the SPL at the source corresponds to a refractive index that is higher as the SPL increases. This finding is in agreement with the results obtained 
in Figure 4, where the non-linear trend between the two variables is evident, particularly above $260 \mathrm{~dB}$ re $1 \mu \mathrm{Pa}$. It is worth noting that a standing wave pressure level will oscillate from zero to a peak midway between the acoustic source and the acoustic mirror. So, even if the location of the peak remain spatially constant over time, the optimal waveguide effect will likewise fluctuate in time due to the change in amplitude. Hence it is necessary to synchronize the optical beam with the acoustic beating in order to optimise the guiding effect given by a UWAOW.

\subsection{UWAOW equation}

In order to evaluate SONAR system performances, based on energy conservation, the sum of signal and gain must be higher than the sum of the detection threshold, transmision losses and noise. ${ }^{2,11}$ Various acoustic signals are present underwater due to many different sources: tides, turbulence, shipping traffic, industrial noise among others. However, the spectrum intensity of these ambient noises is negligible respect to the SPL discussed in this study. Additionally, a system filtering may be used at the receiver's end to reduce the ambient noise influence. An exception may be made when considering few particularly intense construction activities of offshore and near-shore facilites as investigated by Harland et al. ${ }^{32}$ Therefore, in general ambient noise need not be taken into account since the objective of this study is the application of the acousto-optic effect for underwater optical communications and not acoustic ones. Thus the aforementioned relation can be used as a starting point in order to better describe the feasibility of an UWAOW. Considering an acoustic source $S L$ in a scenario with transmission loss $T L$

$$
S L-T L+D I \geq U W A O W,
$$

where $D I$ is the directivity index that results from the angular distribution of the beam pattern and the UWAOW is the amount of SPL (in $\mathrm{dB}$ re $1 \mathrm{\mu Pa}$ ) necessary to obtain the required $R I$ along the communication channel. Various equations are available for $D I$, depending on the transducer geometry. As example, a circular disc transducer array with a diameter $D$, illustrated in Figure 5, is described by the Equation (14a)

$$
D I=\left\{\begin{array}{l}
20 \log _{10}\left(\frac{\pi D}{\lambda_{a c}}\right) \\
10 \log _{10}\left(\frac{L_{x} L_{y}}{\lambda_{a c}^{2}}\right) .
\end{array}\right.
$$

For a rectangular aperture, the $D I$ is given by Equation (14b). In both cases, DI depends on the acoustic frequency chosen for the UWAOW. Thus, in order to have an additive contribution from this term in the overall performance, it is convenient to use a diameter much larger than the acoustic wavelength. The comparison of the Rayleigh distance $z_{0}$ (Equation (11)) and the $D I$ as function of different frequencies and for transducer diameters is given in Figure 7. This finding shows the necessary design trade-offs between the two quantities, since a low acoustic frequency $(f \leq 100 \mathrm{~Hz})$ results in a long Rayleigh distance $z_{0}$ and in a long fundamental acoustic wavelength $\left(\lambda_{a c}^{0} \geq 15 \mathrm{~m}\right)$. This, in turn, requires a rather large transducer diameter $(\geq 2 \mathrm{~m})$ in order to have a positive $D I$ that would direct the acoustic field over a narrow angle and extend the range of the TIR zone.

\subsection{Equal-area annular transducer array}

As briefly discussed in Section 2.5, in practice a Bessel-like beam shows a narrow central lobe with a high intensity and little lateral dispersion up to a certain distance. Over this range, the peak intensity is kept constant as a result of the non-diffractive property arising from the cylindrical coordinates. The distance over which the intense central core will show a non-diffractive behaviour is referred to either as Depth Of Field $\left(\mathrm{DOF}_{B}\right)$ or as propagation distance ${ }^{14,19}$

$$
D O F_{B}=R \sqrt{\left(\frac{k}{\alpha}\right)^{2}-1} .
$$

Up to this distance, the on-axis beam amplitude will obviously oscillate due to its wave nature. When the value of $\mathrm{DOF}_{B}$ is reached, it will fall off quickly. The optimal value of $\mathrm{DOF}_{B}$ depends on the application of the UOCS and it can be estimated by adjusting the annular transducer array parameters. Indeed, $k$ is a function 

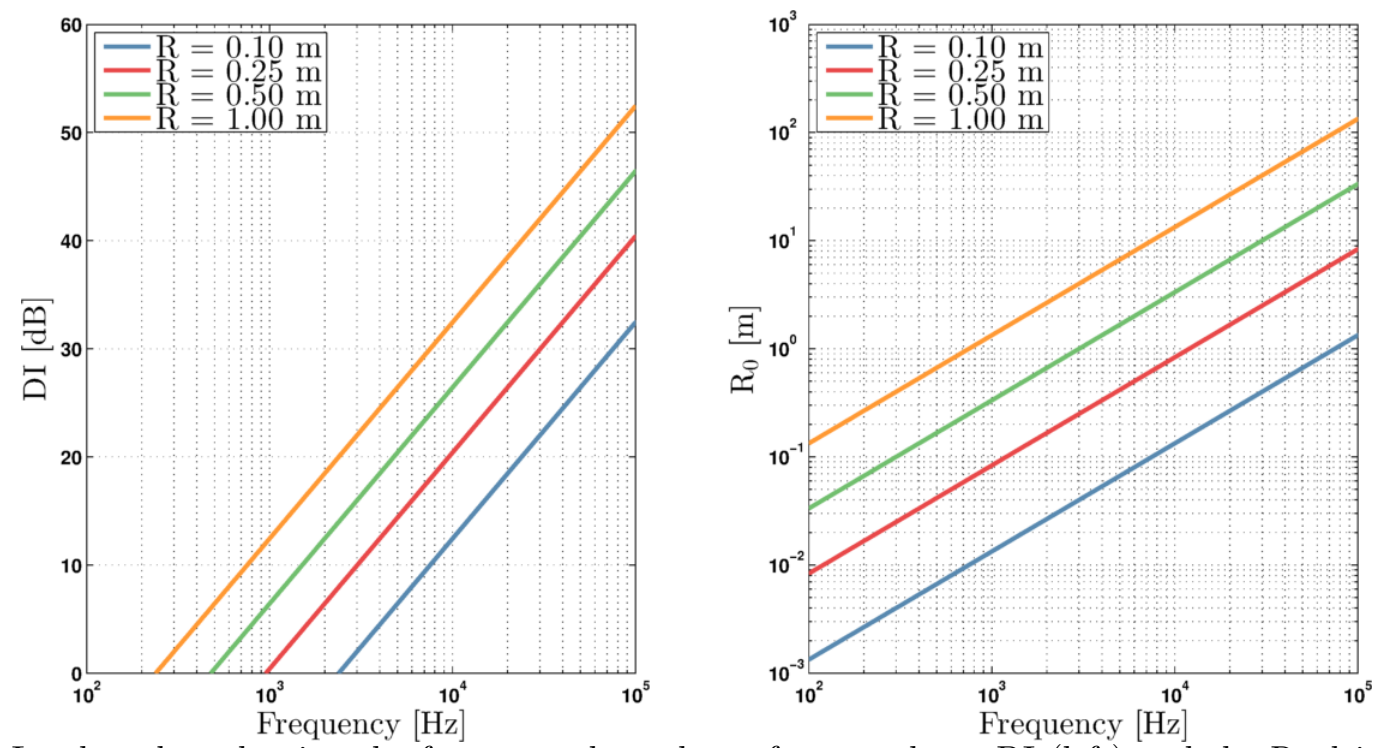

Figure 7: Log-log plots showing the frequency dependence for transducer DI (left) and the Rayleigh distance with four different transducer diameters (right). In the left hand plot, frequency values that result in a negative DI are not shown.

of the acoustic frequency and $\alpha$ (hence $\beta$ ) is given by geometrical factors chosen in the transducer design. The approximation of Bessel beams with an annular transducer array with a total radius $R$ and made up of $N$ element has been comprehensively investigated by Holm, ${ }^{19}$ leading to

$$
\alpha=2.405 \frac{\sqrt{N}}{R} .
$$

Moreover, the choice of equal-area transducer gives the advantage of elements with same phase error and electrical impedance. ${ }^{19}$ The second geometry proposed in this work is illustrated in Figure 8 where an annular transducer array with $N=7$ elements and total diameter $D$. Given the high directionalty and well collimated properties of a Bessel-like beam, an acoustic mirror is not required in this configuration since there is no need for a standing wave. Moreover, the reduced beam spreading up to a given propagation-invariant distance ensures that the SPL at the transducer surface $(z=0)$ will remain substantially unchanged along the communication channel. As highlighted by J. Durnin, ${ }^{14}$ the value of the parameter $\alpha$ has to fall within the wavenumber $k$ and $2 \pi / R$. As a matter of fact, when $\alpha>k$, the $\mathrm{DOF}_{B}$ is zero, as can be quickly checked from Equation (15). On the other hand, if $\alpha<2 \pi / R$ the $\mathrm{DOF}_{B}$ coincides with the conventional Rayleigh range $\mathrm{z}_{R}$ of a Gaussian beam.

The effect of an increasing number of elements, not shown here, in the annular transducer design on the resulting acoustic pattern is an increased number of side lobes, although with a much smaller amplitude than the main one. This finding is in agreement with what one would expect in order to generate a narrow and wellcollimated UWAOW. The range over which the Bessel-like beam remains focused is defined by the $\mathrm{DOF}_{B}$. In the region beyond this value, there is a geometrical shadow zone due to the finite aperture of the transducer. The top view of the normalised sound pressures, reported in Figure 9, shows a set of radially simmetric rings which are analogous to the zone plate in the optical domain, since the equations describing the physics phenomena in the two domains have the wave nature in common. The main feature of the Bessel-like beam is evident, i.e. its hollow profile. The separation between the different rings, corresponding the lobes of the Bessel function, is inversely proportional to $\alpha^{19}$

$$
\rho_{0-0}=\frac{4.81}{\alpha} .
$$

Thus, from Equation (16) and Equation (17), it follows that there will be a lower density of zeros of a Bessel-like beam for a low number of elements $N$ and for a larger radius $R$ of the transducer. These findings are intuitive but due to the boundary condition set in Equation (6) the best hollow beam will be achieved consequently with 


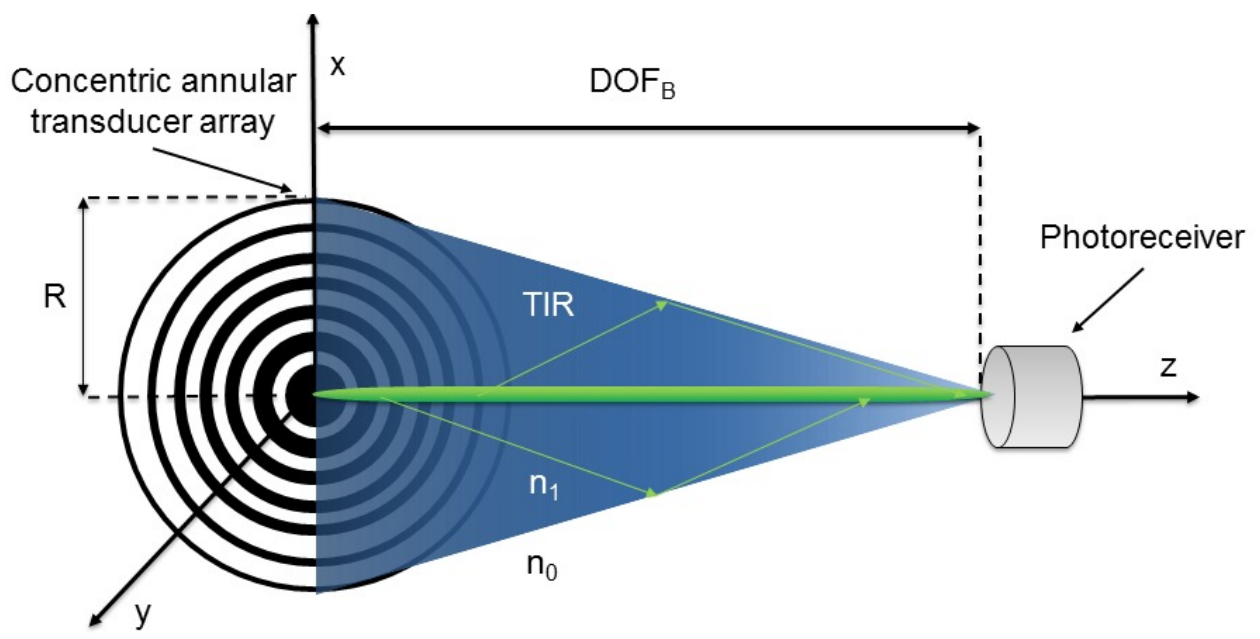

Figure 8: Schematic of the concentric annular transducer array with $N=7$ and radius $R$ that generates an acoustic Bessel-like beam. This achieves the condition for TIR cone that optically encloses and guides the laser beam as well as the scattered photons.

a number of element equal or greater than seven and for large transducer radius. The latter will also extend the $\mathrm{DOF}_{B}$, as previously discussed. Another important feature of the Bessel-like beam is its lateral resolution, $\mathrm{LR}_{B}$, which is set by the bandwidth (FWHM or $\left.-6 \mathrm{~dB}\right)$ as $^{18}$

$$
L R_{B}=\frac{3.04}{\alpha}
$$

In this case, $\mathrm{LR}_{B}$ can be considered as the extension over which the acoustic pressure will actively guide the photons, i.e. the diameter of the waveguide. It is important to note that if the SPL is strong enough to generate a refractive index change, in principle there will still be a guiding effect also beyond the $\mathrm{LR}_{B}$ value. Intuitively, the higher the original SPL and the wider the extension of the UWAOW. Various combinations of radius $R$ and ultrasonic frequencies with $N=7$ elements are listed in Table 6.

\begin{tabular}{|c|c|c|c|c|c|c|c|}
\hline & \multirow{2}{*}{$\mathbf{R}[\mathrm{m}]$} & \multicolumn{3}{|c|}{$\mathrm{DOF}_{B}[\mathbf{m}]$} & \multirow{2}{*}{$\begin{array}{l}\mathbf{2} \pi / \mathbf{R} \\
{\left[\mathbf{m}^{-1}\right]}\end{array}$} & \multirow{2}{*}{$\begin{array}{c}\alpha \\
{\left[\mathbf{m}^{-1}\right]}\end{array}$} & \multirow{2}{*}{$\begin{array}{r}\mathbf{L R}_{B} \\
{[\mathbf{m}]}\end{array}$} \\
\hline & & 20 & 50 & 100 & & & \\
\hline \multirow{4}{*}{$\mathrm{N}=7$} & 0.10 & 0.09 & 0.31 & 0.65 & 62.83 & 63.63 & 0.05 \\
\hline & 0.25 & 0.78 & 2.04 & 4.11 & 25.13 & 25.45 & 0.12 \\
\hline & 0.50 & 3.25 & 8.21 & 16.45 & 12.57 & 12.73 & 0.24 \\
\hline & 1.00 & 13.13 & 32.90 & 65.82 & 6.28 & 6.36 & 0.48 \\
\hline
\end{tabular}

Table 6: Depth of field for an annular transducer array with $N=7$ as a function of total radius $R$ and frequency $f$. The values of the parameters $2 \pi / R, \alpha$ and $L_{B}$ are also reported.

The last columns in the table show that the condition $\alpha>2 \pi / R$ is met only for $N \geqslant 7$, in agreement with the conclusion from Holm, ${ }^{19}$ regardless of the acoustic frequency chosen. The latter set the value of the wavenumber $k$ and thus it has a great influence on the achievable $\mathrm{DOF}_{B}$ as can be noticed in Equation (15) and in the plots in Figure 10. Moreover, the influence of $N$ and $R$ on the lateral resolution is reported in the last column. By combining Equation (6) and Equation (16), it can be noticed that the $\mathrm{DOF}_{B}$ is directly proportional to the square of the radius $R$ whereas it varies linearly with the acoustic frequency. These findings are numerically summarised in Table 6 . It follows that there will be a longer propagation distance by using, when possible, a larger annular transducer array than a higher acoustic frequency. Obviously, if the transducer radius can not be increased any further, an increase in the acoustic frequency will still improve the propagation-invariant distance and thus the communication range over which the UWAOW can be successfully implemented. 

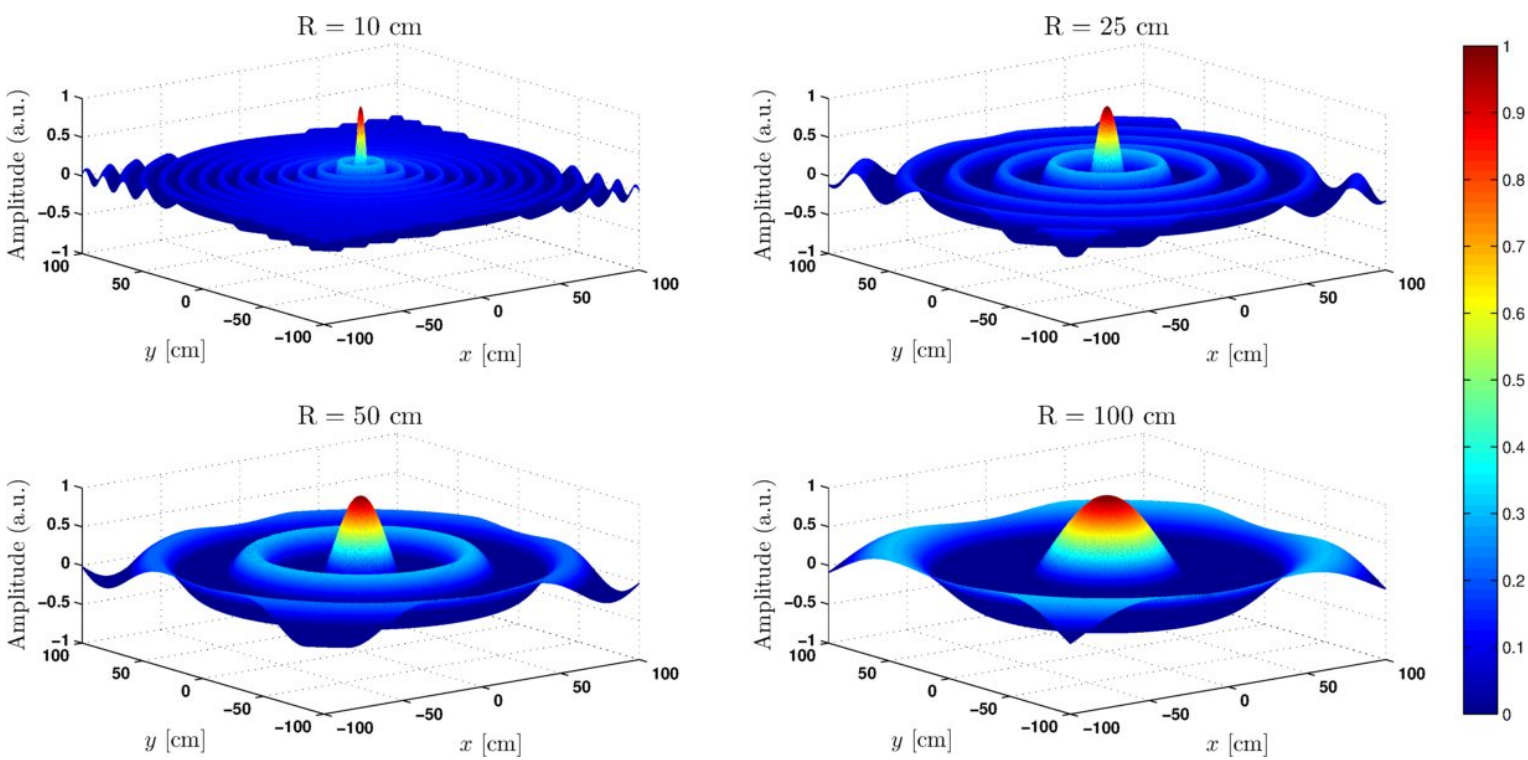

(a) Surface plot
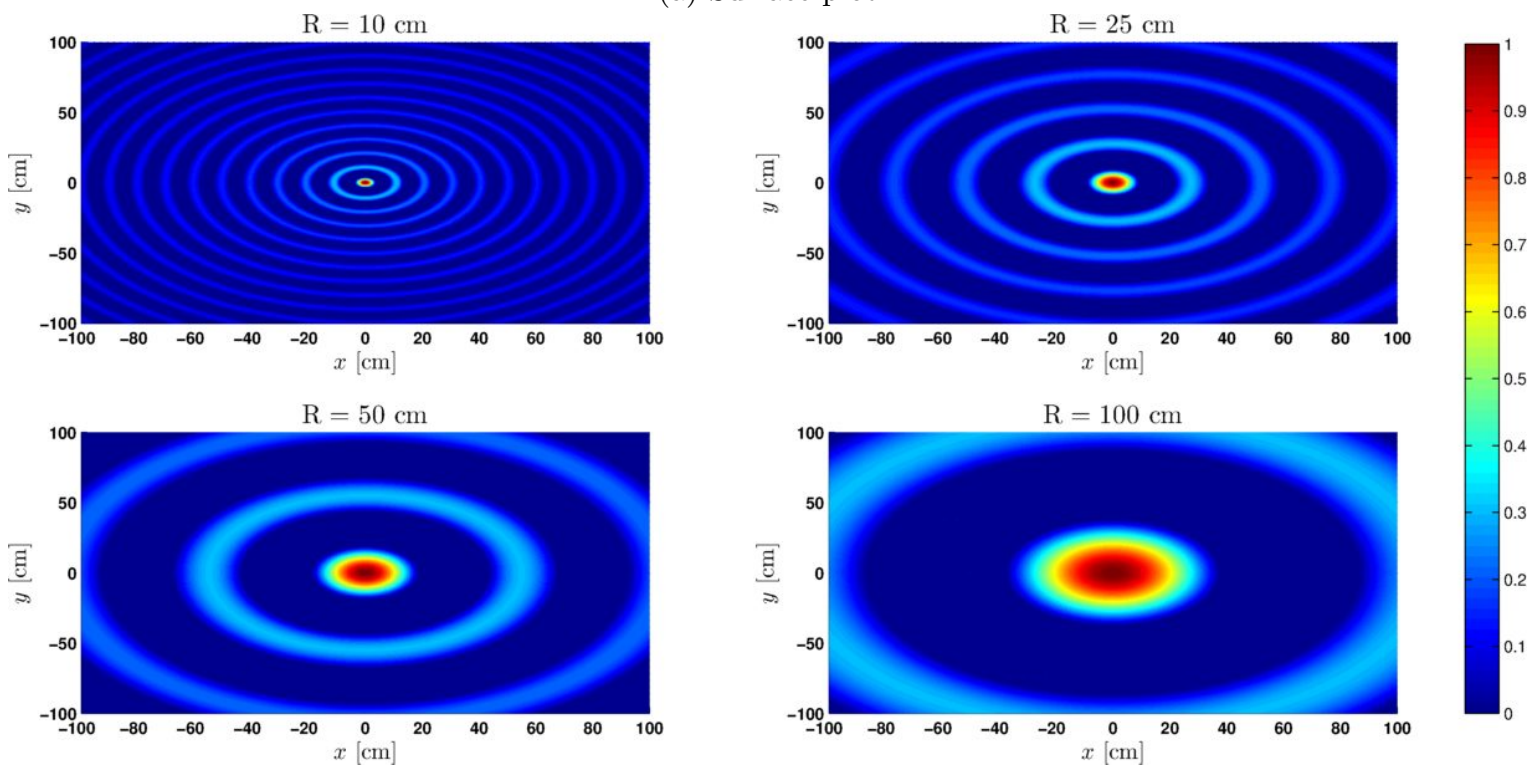

(b) Top view

Figure 9: Surface and top view of the normalised sound pressure at $z=0$ for different transducer radius $R$ with $N=7$ elements.

\subsection{Possible issues related to a high SPL}

The required acoustic field to generate an UWAOW is relatively high compared with normal seismic surveys and SONAR operations. Therefore, a number of technical issues may arise and should be taken into consideration during the UWAOW design process. The first aspect is the mechanical stress to which the materials are subjected, in particular the design of the optical windows in front of the receiver. Ordinary materials (BK7 glass, Silica glass, KRS5) used in optics have elastic limits in the range $10 \mathrm{MPa}$ to $100 \mathrm{MPa}$ ( $\equiv 260 \mathrm{~dB}$ to $280 \mathrm{~dB}$ re $1 \mathrm{\mu Pa}) .{ }^{33-35}$ As discussed in the previous sections, this range corresponds also to the operational one for a UWAOW. Thus a particularly hard crystal is required in the system, such as Sapphire $\left(\mathrm{Al}_{2} \mathrm{O}_{3}\right)$ with an elastic limit between $276 \mathrm{MPa}(288.8 \mathrm{~dB} \text { re } 1 \mu \mathrm{Pa})^{33}$ and $488 \mathrm{MPa}(293.7 \mathrm{~dB}$ re $\left.1 \mu \mathrm{Pa})\right)^{34,35}$ An associated effect arising from such a 

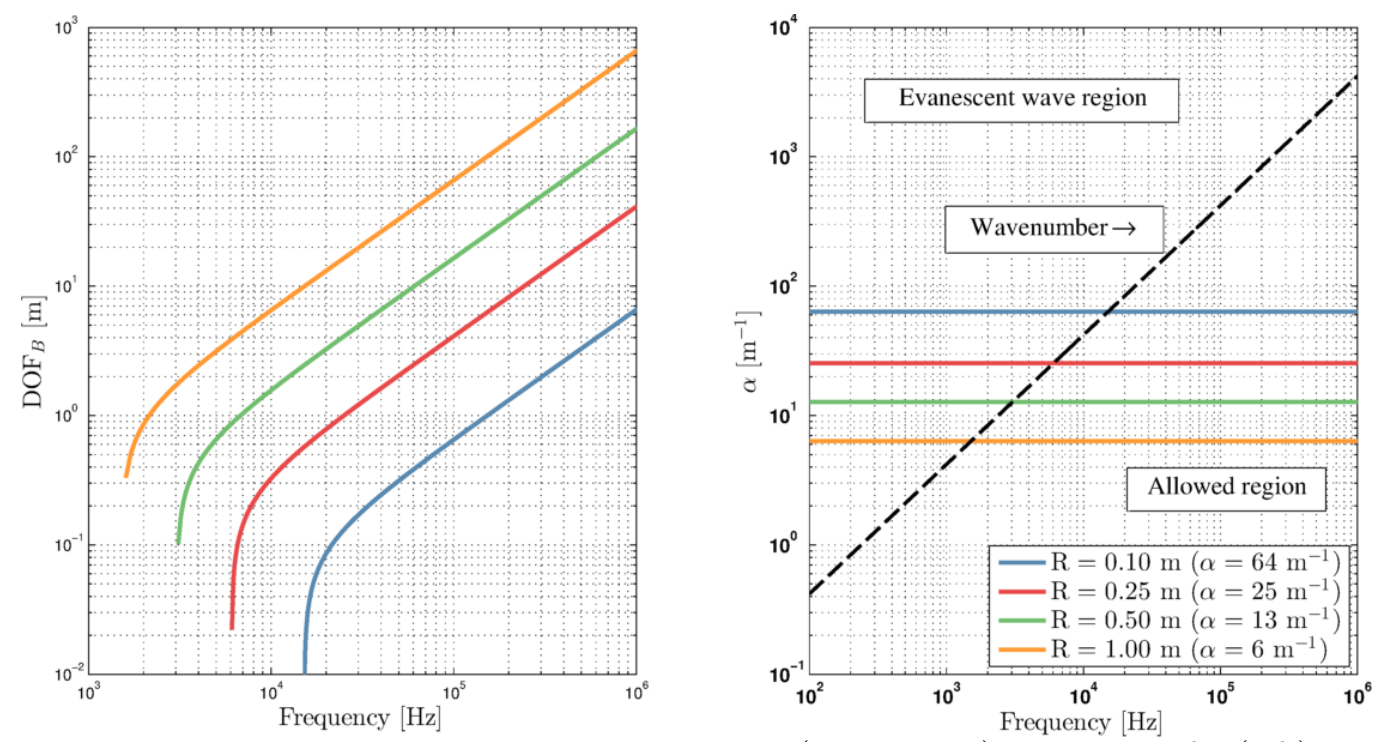

Figure 10: Log-log plots showing the annular transducer radius (with $N=7$ ) dependence for (left) the $\mathrm{DOF}_{B}$ and (right) the parameter $\alpha$. In the latter the black dashed line is the plot of the wavenumber which is independent from the radius and sets an upper limit to the maximum allowed value for $\alpha$.

high SPL is the local increase in temperature due to the high compression of the water. Considering the complex pressure-temperature phase diagram of water, ${ }^{36}$ it can be seen that the solid-liquid phase transition occurs at a pressure of $632.4 \mathrm{MPa}(\equiv 296 \mathrm{~dB}$ re $1 \mu \mathrm{Pa})$ at $-0.16{ }^{\circ} \mathrm{C}(272.99 \mathrm{~K})$. Following the line of equilibrium along its positive slope, the required pressure for this transition rises up to $1 \mathrm{GPa}(\equiv 300 \mathrm{~dB}$ re $1 \mu \mathrm{Pa})$ at $+26.85^{\circ} \mathrm{C}$ $(300 \mathrm{~K})$. From these findings, it is reasonable to avoid such high values of SPL in front of the acoustic source in order to remove the risk of ice formation. Seismic sampling techniques use source levels produced by airgun arrays with SPL $>220 \mathrm{~dB}$ re $1 \mu \mathrm{Pa} @ 1 \mathrm{~m}$, measured zero-to-peak. The acoustic modelling study presented by MacGillivray in 2006 using the JASCO Applied Sciences airgun model reports a peak SPL of $228 \mathrm{~dB}$ re $1 \mu \mathrm{Pa}$ @ $1 \mathrm{~m}$ for a 1 Generator-Injector (GI) airgun (45 $\mathrm{in}^{3}$ generator). ${ }^{37}$ The SPL produced by a single airgun is in the range from $220 \mathrm{~dB}$ to $238 \mathrm{~dB}$ re $1 \mu \mathrm{Pa} @ 1 \mathrm{~m}$ and is non-directional. In order to restrict the angular distribution of the acoustic field, an array of airguns is often employed and SPL up to $265 \mathrm{~dB}$ re $1 \mu \mathrm{Pa} @ 1 \mathrm{~m}$ can be achieved. ${ }^{38,39}$

Another important factor that could arise from a high SPL underwater, is the biological impact of this technology due to the potential stressor on the marine ecosystem. Various models have been developed to evaluate the intense man-made ocean noise (in particular, offshore construction and ship traffic) that could negatively affect marine fauna species in that area. In both the embodiments proposed here, a feasible precaution to mitigate the negative impact of high SPL on the animals would be to gradually increase the acoustic source instead of switching the transducer on directly at the target power. In this way the UWAOW would act as a deterrent for them to get closer to the system. Finally, the main limitation to an endless increase in the acoustic pressure $P_{a c}$ is set by the ambient pressure of the underwater environment $P_{w}$. When the former exceeds the latter, cavitation occurs which generates air bubbles in front of the acoustic source. The source level at which cavitation will occur, $S L_{c a v}$, is increasing with the surface area $S_{t}\left(\mathrm{in}^{2}\right)$ of the transducer and with the depth $z$ (in $\mathrm{m}$ ) since in deeper water the hydrostatic pressure is higher. The cavitation level is described by ${ }^{40}$

$$
S L_{\text {cav }}=186+10 \log \left(S_{t}\right)+D I+20 \log (10+z) .
$$

This equation is plotted in Figure 11 for four acoustic frequencies, considering a disc array of transducers with increasing diameter and up to a depth of $150 \mathrm{~m}$. The results show that in order to avoid cavitation, or at least to minimise it, a large source diameter is necessary. Likewise, a higher acoustic frequency entails a shorter acoustic wavelength $\lambda_{a c}$, thus a higher $D I$ (Equation (14)) as well as a higher $S L_{c a v}$ that would be beneficial to the feasibility of a UWAOW. Indeed, cavitation would strongly scatter the optical beam and lead to a reduction 
in the SPL, thus this phenomenon will need to be addressed in order to be able to take full advantage of the UWAOW technology.
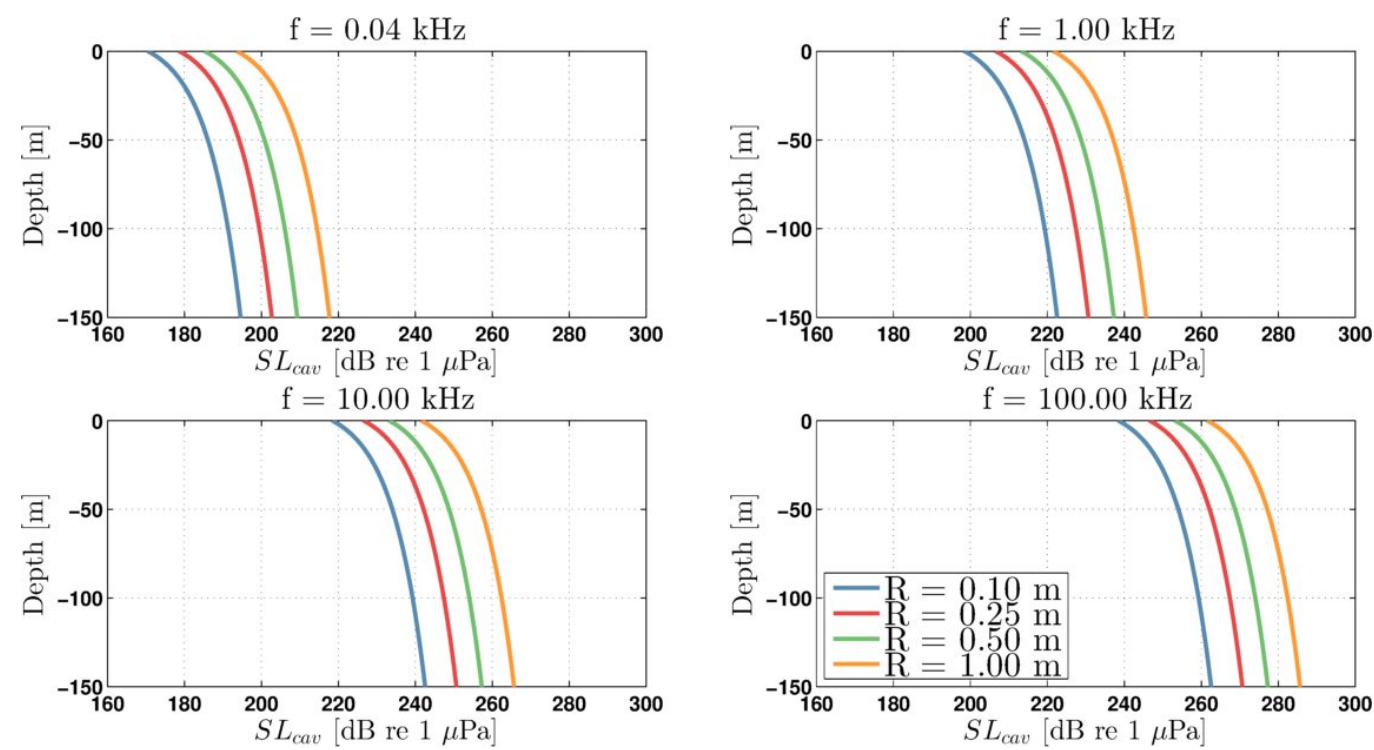

Figure 11: Cavitation level as a function of the depth at which the system is operating for five transducer diameters at four acoustic frequencies.

\subsection{Worked examples}

The required SPL depends on the desired refractive index contrast, on the geometry of the propagation regime and on the communication range that is necessary to establish. Given all the previously discussed points, two numerical examples are presented with an arbitrarily chosen value of the refractive index of $\simeq 0.3 \%$ in both configurations.

\subsubsection{Standing wave}

When selecting the geometry proposed in Section 3.1, due to the spherical spreading of the acoustic beam, an estimated SPL value of about $280 \mathrm{~dB}$ re $1 \mathrm{\mu Pa}$ (equivalent to a voltage applied to the transducer in excess of $5 \mathrm{kV}$ ) would be required for a link up to $20 \mathrm{~m}$. Thus, for a link of $20 \mathrm{~m}$, an acoustic wavelength of $40 \mathrm{~m}$ is required. Hence the acoustic frequency has to be set to the fundamental harmonic $f_{0}=c_{w} / \lambda_{a c}=1500 \mathrm{~m} \mathrm{~s}^{-1} / 40 \mathrm{~m}=37.5 \mathrm{~Hz}$. It is understood that in order to achieve this result, the operational communication link length must be known. Moreover, in a dynamic system the instantaneous length of the UWAOW (ideally equal to $\lambda_{a c} / 2$ ) can be optimised by adjusting either the receiver as a whole or the acoustic mirror only. In this worked example cavitation effects are not taken into account, since with such a low frequency the required transducer diameter would be not practical to realise, even for a system operating in deep water, as plotted in the top left panel in Figure 11 for $f=40 \mathrm{~Hz}$.

\subsubsection{Annular transducer array}

By adopting the geometry presented in Section 3.4, the amount of SPL required is lower than the previous geometry and an acoustic mirror is no longer required. This results from the propagation-invariant nature of a Bessel-like beam that is able to maintain the necessary refractive index along the communication range up to the $\mathrm{DOF}_{B}$ (see Table 6). In order to obtain an arbitrarily chosen value for $\mathrm{RI} \simeq 0.3 \%$ (see Table 5 ) it is necessary a SPL of about $270 \mathrm{~dB}$ re $1 \mu \mathrm{Pa}$, thus about $10 \mathrm{~dB}$ less than with a standing wave approach. One drawback of choosing higher acoustic frequencies is that there will be a non-negligible impact of the medium. From the values for $\alpha^{\prime}$ reported in Table 2, the sound absorption in seawater at $100 \mathrm{kHz}$ over a link of $30 \mathrm{~m}$ can be evaluated as approximately $1 \mathrm{~dB}$. This gain can be seen as an analogy for the directivity index $D I$ in the UWAOW Equation (13), since it arises from the property of a Bessel-like beam in being well-collimated and 
diffraction-free up to a given distance that results in an efficiently allocated sound pressure. The latter can be determined by using Equation (15) of which some numerical values are listed in see Table 6. For example, a radius of the annular transducer array of $0.5 \mathrm{~m}$ and a $f=100 \mathrm{kHz}$ will result in a $\mathrm{DOF}_{B}=16.45 \mathrm{~m}$. On the other hand, doubling the radius $(R=1.0 \mathrm{~m})$ and halving the acoustic frequency $(f=50 \mathrm{kHz})$ will extend the range up to $32.90 \mathrm{~m}$. Thanks to the higher order of magnitude of the acoustic frequency compared to the previous geometry $(\mathrm{kHz}$ vs $\mathrm{Hz})$, there is an advantageous and substantial increase in the cavitation level. As it can be seen in bottom right panel in Figure 11, with $R=0.5 \mathrm{~m}(R=1.0 \mathrm{~m}), \mathrm{SL}_{\text {cav }}>270 \mathrm{~dB}$ re $1 \mu \mathrm{Pa}$ from a depth of $59 \mathrm{~m}(16 \mathrm{~m})$. These values are slightly overestimated since the total radius of the annular transducer array does not take into account the spacing between the different elements. Hence, the actual surface area of the acoustic source $\left(S_{t}\right.$ in Equation (19) will be smaller than a disc with the same radius. It is worth noting that an higher acoustic frequency will entail a longer $\mathrm{DOF}_{B}$ along with reduced cavitation problems, which are depth dependent. The lower SPL required results in a less challenging, albeit not straightforward, engineering task, as well as a low probability of ice formation on the surface of the transducer and a shorten minimum range safety.

In comparison with the results from the previous worked example, the practical advantage is evident. Comparative plots illustrating the influence of the annular transducer radius on the resulting refractive index of perturbed seawater $\left(n_{1}\right)$ at $z=0$ with a SPL $=270 \mathrm{~dB}$ re $1 \mu \mathrm{Pa}$ are shown in Figure 12 for a radial distance of \pm $0.1 \mathrm{~m}$ along the $x$ - and $y$ - axes. It can be noticed the narrow region over which the sound pressure exerted by the acoustic Bessel-like beam results in an a localised and well defined increase in the refractive index of water from the unperturbed value of $n_{0}=1.334344$ as given by from IAPWS at $589 \mathrm{~nm} .{ }^{27}$
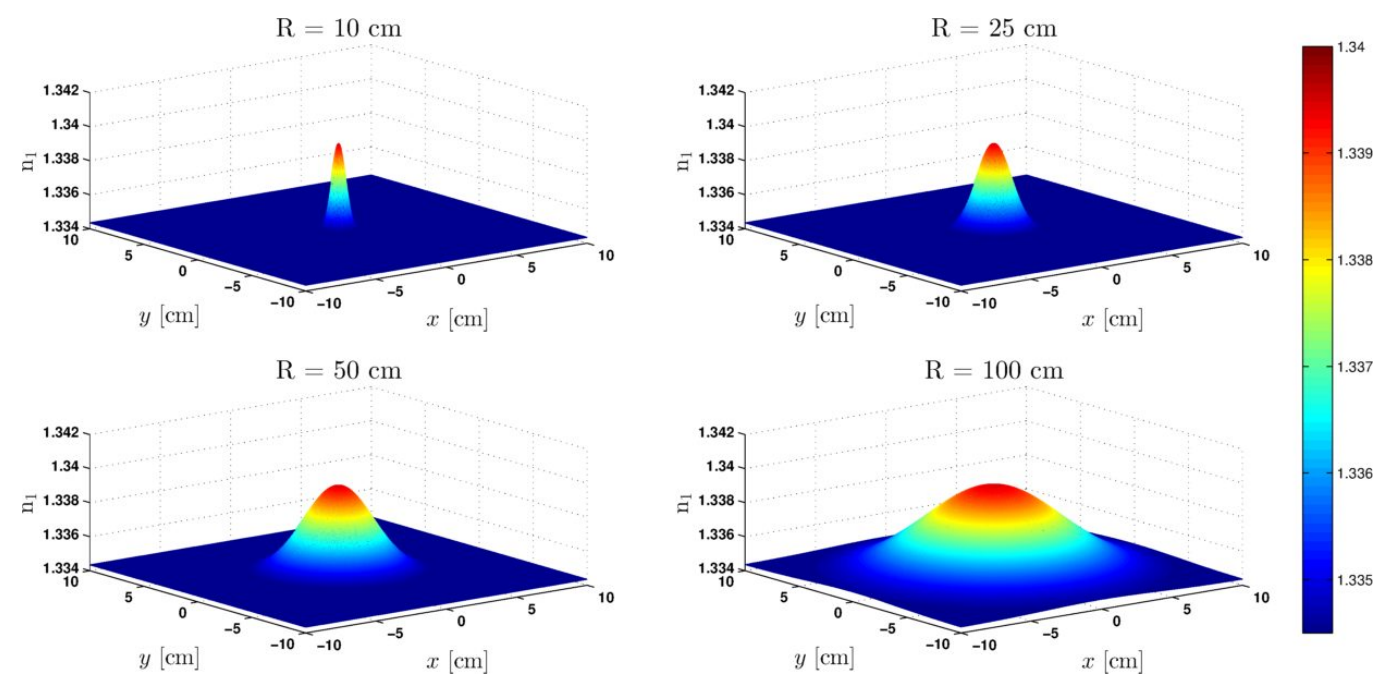

Figure 12: Refractive index of seawater at $z=0$ with a $\mathrm{SPL}=270 \mathrm{~dB}$ re $1 \mu \mathrm{Pa}$ as a function of the annular transducer radius.

\section{CONCLUSIONS}

Two different geometries have been presented, one operating in the low range of the acoustic frequency spectrum and the second at ultrasonic frequencies, with regards to the implementation of an UWAOW in an underwater free-space optical link. The associated implementation issues have been also analysed and summarised. The potentially advantage in reducing the medium scattering by coupling the optical beam to the waveguide, that would confine the launched photons resulting in minimised the losses due to the medium, has been discussed. Hence this approach is expected to augment the received optical power, much to the advantage of system link budget. It is understood that in order to generate the SPLs necessary to obtain an UWAOW high power consumption devices are required. Thus, this kind of technology is unlikely to be employed as a portable system. However, it would be possible to overcome these drawbacks by taking advantage of the existing technology already on board current ships and submarines. These have indeed the full capabilities to deal with a relatively heavy payload and added complexity given by the implementation of an UWAOW. Since acoustic sources are 
a widely and mature technology in underwater communication, the technique presented in this study could be integrated with standard active SONAR systems as scanning and tracking beams. These could be used to align the transmitter and the receiver in the initial link acquisition and thereafter also to maintain the alignment throughout the whole communication process. Thus, an UWAOW can be also envisioned as a valid tool in any pointing and tracking system. The proposed novel technique in this work will hopefully stimulate interest in further research and deeper investigations in what, given the present state-of-the-art of technology, might be envisioned as a thought experiment only.

\section{Acknowledgments}

The authors wish to thank the Engineering and Physical Sciences Research Council (EPSRC reference 1566934) and BAE Systems Plc. for sponsorship of this work. The results of this work have been included in the UK Patent Application No.GB 1704940.4 and European Patent Application No. EP 17275042.4, both filed on 28 March 2017.

\section{REFERENCES}

[1] Mobley, C. D., [Light and water: radiative transfer in natural waters], Academic press (1994).

[2] Urick, R., "Principles of underwater acoustics," (1983).

[3] Jack, S. H., Hann, D. B., and Greated, C. A., "Influence of the acousto-optic effect on laser Doppler anemometry signals," Review of scientific instruments 69(12), 4074-4081 (1998).

[4] Buick, J., Cosgrove, J., Douissard, P.-A., Greated, C., and Gilabert, B., "Application of the acousto-optic effect to pressure measurements in ultrasound fields in water using a laser vibrometer," Review of scientific instruments 75(10), 3203-3207 (2004).

[5] Enayet, M., Gibson, M., Taylor, A., and Yianneskis, M., "Laser-Doppler measurements of laminar and turbulent flow in a pipe bend," International Journal of Heat and Fluid Flow 3(4), 213-219 (1982).

[6] Herzog, P., Valiere, J., Valeau, V., and Tournois, G., "Acoustic velocity measurements by means of laser Doppler velocimetry," in [8th Intl. Symp. On Appl. Of Laser Techniques to Fluid Mechanics, July, 8th-11th, Lisbon Portugal], (1996).

[7] Loizeau, T. and Gervais, Y., "Measurement of the acoustic velocity by laser Doppler anemometry," Acta Acustica united with Acustica 83(6), 945-954 (1997).

[8] Ikezoe, Y., Hirota, N., Nakagawa, J., and Kitazawa, K., "Making water levitate," Nature 393(6687), 749-750 (1998).

[9] Hosoda, H., Mori, H., Sogoshi, N., Nagasawa, A., and Nakabayashi, S., "Refractive indices of water and aqueous electrolyte solutions under high magnetic fields," The Journal of Physical Chemistry A 108(9), 1461-1464 (2004).

[10] Fisher, F. and Simmons, V., "Sound absorption in sea water," The Journal of the Acoustical Society of America 62(3), 558-564 (1977).

[11] Jensen, F. B., Kuperman, W. A., Porter, M. B., and Schmidt, H., [Computational ocean acoustics], Springer Science \& Business Media (2000).

[12] Leroy, C. C., Robinson, S. P., and Goldsmith, M. J., "A new equation for the accurate calculation of sound speed in all oceans," The Journal of the Acoustical Society of America 124(5), 2774-2782 (2008).

[13] Giuliano, G., Viola, S., Watson, S., Laycock, L., Rowe, D., and Kelly, A. E., "Laser based underwater communication systems," in [Transparent Optical Networks (ICTON), 2016 18th International Conference on], 1-4, IEEE (2016).

[14] Durnin, J., "Exact solutions for nondiffracting beams. I. The scalar theory," JOSA A 4(4), 651-654 (1987).

[15] Laycock, L. and Webster, S., "Bessel beams-their generation and application," GEC Journal Research 10, 36-51 (1992).

[16] McQueen, C., Arlt, J., and Dholakia, K., "An experiment to study a nondiffracting light beam," American Journal of Physics 67(10), 912-915 (1999).

[17] Arlt, J. and Dholakia, K., "Generation of high-order Bessel beams by use of an axicon," Optics Communications 177(1), 297-301 (2000). 
[18] Lu, J.-Y. and Greenleaf, J. F., "Ultrasonic nondiffracting transducer for medical imaging," IEEE transactions on ultrasonics, ferroelectrics, and frequency control 37(5), 438-447 (1990).

[19] Holm, S., "Bessel and conical beams and approximation with annular arrays," IEEE transactions on ultrasonics, ferroelectrics, and frequency control 45(3), 712-718 (1998).

[20] Masuyama, H., Yokoyama, T., Nagai, K., and Mizutani, K., "Generation of Bessel beam from equiamplitudedriven annular transducer array consisting of a few elements," Japanese journal of applied physics 38(5S), 3080 (1999).

[21] Rhodes, D., Gherardi, D., Livesey, J., McGloin, D., Melville, H., Freegarde, T., and Dholakia, K., "Atom guiding along high order Laguerre-Gaussian light beams formed by spatial light modulation," Journal of modern optics 53(4), 547-556 (2006).

[22] Oil and Gas Producers (OGP), "Fundamentals of underwater sound," tech. rep., No. 406 (May 2008).

[23] Zhou, Q., Cha, J. H., Huang, Y., Zhang, R., Cao, W., and Shung, K. K., "Alumina/epoxy nanocomposite matching layers for high-frequency ultrasound transducer application," IEEE transactions on ultrasonics, ferroelectrics, and frequency control 56(1), 213-219 (2009).

[24] Zhang, R., Cao, W., Zhou, Q., Cha, J. H., Shung, K. K., and Huang, Y., "Acoustic properties of alumina colloidal/polymer nano-composite film on silicon," IEEE Transactions on Ultrasonics Ferroelectrics and Frequency Control 54(3), 467 (2007).

[25] Schiebener, P., Straub, J., Sengers, J. L., and Gallagher, J., "Refractive index of water and steam as function of wavelength, temperature and density," Journal of physical and chemical reference data 19(3), 677-717 (1990).

[26] Weiss, L., Tazibt, A., Tidu, A., and Aillerie, M., "Water density and polarizability deduced from the refractive index determined by interferometric measurements up to $250 \mathrm{MPa}$," The Journal of Chemical Physics 136(12), 124201 (2012).

[27] Fernández-Prini, R. and Dooley, R., "Release on the refractive index of ordinary water substance as a function of wavelength, temperature and pressure," International Association for the Properties of Water and Steam , 1-7 (1997).

[28] Pinnow, D., "Guide lines for the selection of acoustooptic materials," IEEE Journal of Quantum Electronics 6(4), 223-238 (1970).

[29] Stoemmer, R., "Acoustic mirror," (June 12 2007). US Patent 7,230,509.

[30] Barber, B., Huggins, H., Miller, R., Murphy, D., and Wong, Y., "Acoustic mirror materials for acoustic devices," (Aug. 5 2003). US Patent 6,603,241.

[31] Sherman, C. H. and Butler, J. L., [Transducers and arrays for underwater sound], vol. 4, Springer (2007).

[32] Harland, E., Jones, S., and Clarke, T., "Sea 6 technical report: Underwater ambient noise," A report by QinetiQ as part of the UK Department of Trade and Industrys offshore energy Strategic Environmental Assessment programme (2005).

[33] "The design of pressure windows." www.crystran.co.uk/userfiles/files/design-of-pressure-windows .pdf.

[34] "Optical windows under pressure." http://www.alkor.net/pressure-window.html.

[35] "Design of pressure window." http://www.ispoptics.com/articles/8/DESIGN\%200F\%20PRESSURE\% 20WINDOW.

[36] "Aqueous salt solutions." http://www.phasediagram.dk/invariant_points.htm.

[37] MacGillivray, A. O., An acoustic modelling study of seismic airgun noise in Queen Charlotte Basin, $\mathrm{PhD}$ thesis, University of Victoria (2006).

[38] Richardson, W. J., Greene Jr, C. R., Malme, C. I., and Thomson, D. H., [Marine mammals and noise], Academic press (2013).

[39] Caldwell, J. and Dragoset, W., "A brief overview of seismic air-gun arrays," The leading edge 19(8), 898-902 (2000).

[40] "United States Naval Academy." https://www.usna.edu/Users/physics/ejtuchol/documents/SP411/ Chapter16.pdf. 